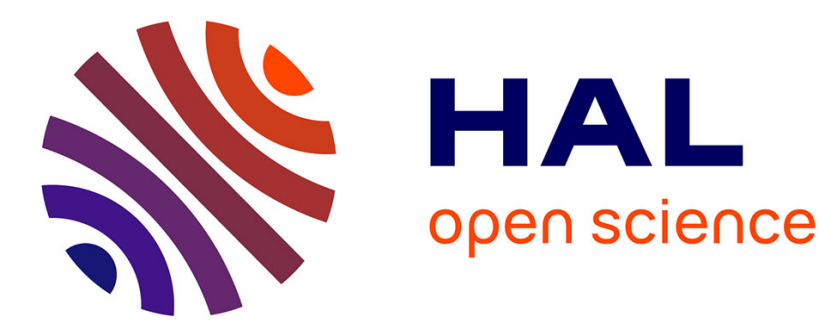

\title{
On the application of the Ritz method to free vibration and buckling analysis of highly anisotropic plates
}

\author{
R. Vescovini, L. Dozio, M. d'Ottavio, O. Polit
}

\section{To cite this version:}

R. Vescovini, L. Dozio, M. d'Ottavio, O. Polit. On the application of the Ritz method to free vibration and buckling analysis of highly anisotropic plates. Composite Structures, 2018, 192, pp.460-474. hal01982525

\section{HAL Id: hal-01982525 \\ https://hal.parisnanterre.fr/hal-01982525}

Submitted on 15 Jan 2019

HAL is a multi-disciplinary open access archive for the deposit and dissemination of scientific research documents, whether they are published or not. The documents may come from teaching and research institutions in France or abroad, or from public or private research centers.
L'archive ouverte pluridisciplinaire HAL, est destinée au dépôt et à la diffusion de documents scientifiques de niveau recherche, publiés ou non, émanant des établissements d'enseignement et de recherche français ou étrangers, des laboratoires publics ou privés. 


\title{
On the application of the Ritz method to free vibration and buckling analysis of highly anisotropic plates
}

\author{
R. Vescovini, L. Dozio, M. D’Ottavio, O. Polit
}

\section{Introduction}

Since its original formulation in 1909 [1], the Ritz method has been largely employed to obtain approximate yet reliable solutions of various structural problems, including plates of different shapes, material properties and boundary conditions.

The ease of formulation, which is especially true for structures characterized by relatively simple domains, in conjunction with the favorable balance of accuracy and number of degrees of freedom, make the method of Ritz an interesting strategy even for this day and age, in particular when extensive parametric and optimization studies are performed during the preliminary stages of the design process. One of the most important steps in the development of Ritz-based models consists of choosing a the set of admissible functions when approximating the problem's unknowns. In the past, several different strategies have been proposed in this regard. Following the seminal work of Ritz [1], who presented the free vibration solution of a completely free rectangular plate using a series of multiplications of free-free beam vibration mode shapes, beam eigenfunctions have been used in many cases to obtain frequency parameters and buckling loads of rectangular plates involving various combinations of boundary constraints [2-4]. However, this procedure may be inappropriate for handling one or more free edges, as observed by Bassily and Dickinson [5], who introduced degenerated beam functions to overcome this restriction. Orthogonal polynomials represent another common set in the Ritz approximation of plate problems. They were first introduced by Bath [6], who discussed the comparison against results obtained with beam characteristic functions [4] and simply-supported plate functions [7]. Different families of orthogonal polynomials were successively used by other researchers $[8,9]$, and sometimes in combination with the GramSchmidt orthogonalization process [10]. Another procedure, conceptually simpler, consists of using ordinary polynomials expansions, where no orthogonalization process is performed. Examples can be found in Refs. [11-13], where different boundary conditions can be handled by proper modification of the trial functions. While different kinds of polynomial expansions were found to have similar convergence properties, they are generally characterized by dissimilar stability properties [14]. Oosterhout et al. [15] observed that simple polynomials can be adopted up to 11 trial functions before becoming unstable. On the contrary, orthogonal polynomials satisfy the stability conditions, consisting in the strong minimality in the energy space of the relevant operator, and higher number of terms in the Ritz series can be used [16].

Another popular choice is given by the expansion in trigonometric series, with restriction to clamped - in this case making use of Lagrange multipliers -, simply-supported and elastically restrained edges 
[17-19]. It is worth noting that this choice is particularly useful when dealing with geometrically nonlinear analyses due to the possibility of obtaining an exact solution of the compatibility equation $[19,20]$. Two decades ago, Beslin and Nicolas [21] proposed a simple yet powerful set of trigonometric functions, which can be readily applied to plates having any combination of edge conditions and offers great numerical stability at higher frequencies. In addition, the set involves simple algebra and calculus and is very efficient from a computational point of view since it can lead to highly sparse eigenvalue problems [22].

A comprehensive recent review regarding the admissible functions used in the Ritz method is available in Ref. [23], where focus is given on thin isotropic plates. Merits and demerits of six sets of functions are discussed and compared in terms of convergence, computational time and numerical stability.

When assessing the global response of isotropic plates in terms of frequency parameters and buckling loads, the effect of different trial functions on the convergence of the solution is relatively inconsequential, at least when the first few eigenvalues are of concern. One of the main advantages of adopting efficient basis, such as orthogonal polynomials, is given by the possibility of accurately capturing higher modes [15]. On the contrary, the analysis of composite plates is more challenging due to a series of complicating effects introduced by the elastic couplings characterizing their constitutive law. Stone and Chandler showed the inadequacy of sine series expansions when applied to the analysis of simply-supported anisotropic thin plates [24]. This inadequacy is mainly associated with bending/twisting coupling effects: the inexact fulfillment of the natural boundary conditions determines the onset of artificial constraints on the plate rotation at the edges, which, in turn, leads to the over-prediction of the eigenvalues. Similar findings were observed in Ref. [25], where sandwich plates with composite faceplates were analyzed using a refined B-spline finite strip approach. In those configurations where elastic coupling effects are stronger, buckling loads computed by the finite strip method are markedly smaller (with differences of the order of 25\%) in comparison with the over-stiff results derived in Ref. $[26,27]$ using trigonometric functions in the Ritz method. The difficulties in the convergence of the Ritz method when applied to anisotropic plates are also discussed in Ref. [28], where it is concluded that results using Ritz method can be unsatisfactory depending on the lay-up and boundary conditions.

Complicating effects are exacerbated by extreme levels of anisotropy, as demonstrated by Wu et al. [29], where the authors, through comparison of different variational approaches, state that the Ritz method suffers from very slow convergence due to the difficulties in satisfying natural boundary conditions and the presence of highly localized deformations close to the boundaries. The application of the method was restricted by the onset of ill-conditioning problems, detected for a number of Legendre-type functions higher than 23.

With regard to the analysis of thick composite plates, efficient Ritzbased approaches were developed in the context of Carrera's Unified Formulation (CUF) for the analysis of simply-supported plates in Ref. [30-33]. Similarly, Fiedler et al. [34] proposed the use of trigonometric functions in plate models based on a generalized higher-order equivalent single layer theory. Within the CUF framework, different sets of boundary conditions were considered by the authors, using polynomial functions [35], Chebyshev [36,37] and Legendre polynomials [38,39].

Despite the vast amount of studies focused on the method of Ritz in the last four decades, it is believed that many aspects associated with its application to the analysis of highly anisotropic thin and thick plates are still unexplored and not covered by the literature. First, the Ritz implementations available in the literature suffer from restrictions in terms of degrees of freedom that can be successfully handled. In the great majority of the cases, 10-20 functions are adopted along the two directions $[28-34,36,37,40]$. This restriction prevents the possibility of obtaining accurate upper-bound predictions for plates characterized by high degree of anisotropy, where several terms are generally necessary. To this aim an efficient implementation is necessary, as the one proposed in this work.

A second aspect of great interest is the evaluation of the effects of different kinds of anisotropy on the convergence properties of the Ritz method. To the best of the authors' knowledge, just a few studies have tackled this topic but, in general, they are restricted to specific cases, and do not provide a comprehensive presentation of the subject involving thick laminates. In addition, no systematic study was found about the effects of different admissible functions on the convergence, accuracy and computational burden of the Ritz method, when applied to thick and moderately thick plates.

The present paper aims at filling these gaps by using a formulation capable of considering several theories, from CLT up to high-order kinematic theories, in a way suitable for studying highly anisotropic thick and moderately thick plates within a unified modeling framework.

\section{Theoretical framework}

This section provides an overview of the Ritz-based variable-kinematic formulation. While further details of this approach can be found in previous works of the authors $[37,41]$, emphasis is here given on the choice among diverse admissible functions and the efficient evaluation of the Ritz in-plane integrals. This latter aspect is a focal point for the subsequent derivation of refined solutions for plates characterized by high degree of anisotropy. It is worth noting that the efficient computation of the Ritz integrals as explained below has been similarly applied to the in-plane integrals arising from CLT-based plate models, which are not reported here for the sake of brevity.

\subsection{Variable-kinematic formulation}

The variable-kinematic formulation is developed in the context of a displacement-based approach, referring to the Principle of Virtual Displacements (PVD). A laminated plate composed of an arbitrary number of plies $N_{l}$ is considered, and a sketch is reported in Fig. 1. The $x$ - and $y$-axis are directed along the longitudinal and transverse directions, respectively, while the $z$-axis is normal to plate midsurface to obtain a right-handed system. The edges are numbered from 1 to 4 in according to the convention reported in the figure, which is adopted next for specifying the boundary conditions.

The variational statement is here expressed with regard to the freevibration and buckling problems. The expression is:

$$
\begin{aligned}
& \sum_{k=1}^{N_{l}} \int_{\Omega} \int_{z k}^{z_{k+1}}\left(\delta \epsilon_{\mathrm{p}}^{k^{T}} \sigma_{\mathrm{p}}^{k}+\delta \epsilon_{\mathrm{n}}^{k^{T}} \sigma_{\mathrm{n}}^{k}+\delta \epsilon_{p_{n l}}^{k^{T}} \sigma_{p 0}^{k}\right) \mathrm{d} z \mathrm{~d} \Omega \\
& =-\sum_{k=1}^{N_{l}} \int_{\Omega} \int_{z k}^{z_{k+1}} \rho^{k} \delta \mathbf{u}^{k^{\mathrm{T}}} \ddot{\mathbf{u}}^{k} \mathrm{~d} z \mathrm{~d} \Omega
\end{aligned}
$$

where the integration is carried out over the domain $\Omega$, defined as $[0 a] \times[0 b]$. The vector $\mathbf{u}^{k}$ collects the three displacement components of the generic ply $k$; the vectors $\epsilon_{\mathrm{p}}^{k}$ and $\epsilon_{\mathrm{n}}^{k}$ are those relative to the inplane and normal components of the small-displacement Green-Lagrange strain tensor, respectively. In a similar fashion, the stress vector $\sigma$ is partitioned into in-plane and normal components $\sigma_{\mathrm{p}}^{k}$ and $\sigma_{\mathrm{n}}^{k}$. In the context of buckling analysis, the additional contributions $\epsilon_{p_{n l}}^{k^{T}}$ and $\sigma_{p 0}^{k}$ are accounted for, representing the nonlinear part of the Green-Lagrange strain tensor and the pre-buckling stress distribution, whose expression can be found in [37].

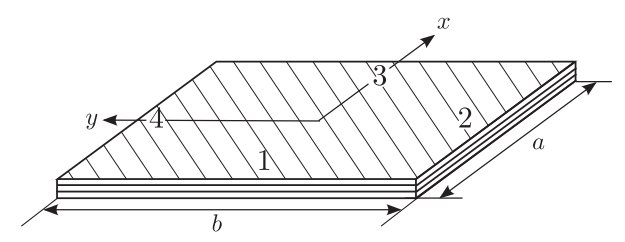

Fig. 1. Laminate with coordinate system, dimensions and numbering of the edges. 
Within the context of the displacement-based approach considered here, the internal virtual work needs to be expressed in terms of the displacement components. This is accomplished by introducing the 3D constitutive law, defined as:

$\sigma_{p}^{k}=\widetilde{\mathbf{C}}_{\mathrm{pp}}^{k} \epsilon_{p}^{k}+\widetilde{\mathbf{C}}_{\mathrm{pn}}^{k} \epsilon_{\mathrm{n}}^{k}$

$\sigma_{n}^{k}=\widetilde{\mathbf{C}}_{\mathrm{pn}}^{k^{\mathrm{T}}} \epsilon_{p}^{k}+\widetilde{\mathbf{C}}_{\mathrm{nn}}^{k} \epsilon_{\mathrm{n}}^{k}$

which leads to the expression:

$$
\begin{aligned}
& \sum_{k=1}^{N_{l}} \int_{\Omega} \int_{z k}^{z_{k+1}}\left\{\left(\mathbf{D}_{\mathrm{p}} \mathbf{u}^{k}\right)^{\mathrm{T}}\left[\widetilde{\mathbf{C}}_{\mathrm{pp}}^{k} \mathbf{D}_{\mathrm{p}} \mathbf{u}^{k}+\widetilde{\mathbf{C}}_{\mathrm{pn}}^{k}\left(\mathbf{D}_{\mathrm{n}} \mathbf{u}^{k}+\mathbf{u}_{, z}^{k}\right)\right]\right. \\
& \left.\quad+\left(\mathbf{D}_{\mathrm{n}} \mathbf{u}^{k}+\mathbf{u}_{z,}^{k}\right)^{\mathrm{T}}\left[\widetilde{\mathbf{C}}_{\mathrm{np}}^{k} \mathbf{D}_{p} \mathbf{u}^{k}+\widetilde{\mathbf{C}}_{\mathrm{nn}}^{k}\left(\mathbf{D}_{\mathrm{n}} \mathbf{u}^{k}+\mathbf{u}_{, z}^{k}\right)\right]+\delta \epsilon_{p_{n l}}^{k^{T}} \sigma_{p 0}^{k}\right\} \mathrm{d} z \mathrm{~d} \Omega \\
& \quad-\sum_{k=1}^{N_{l}} \int_{\Omega} \int_{z k}^{z_{k+1}} \rho^{k} \delta \mathbf{u}^{k^{\mathrm{T}}} \ddot{\mathbf{u}}{ }^{k} \mathrm{~d} z \mathrm{~d} \Omega
\end{aligned}
$$

where the terms $\mathbf{D}_{n}$ and $\mathbf{D}_{p}$ are matrices of differential operators defining the strain-displacement relations that are detailed in Ref. [37].

The underlying kinematic theory adopted here refers to the Generalized Unified Formulation (GUF), proposed by Demasi [42,43], representing an extension of the well-known Carrera's Unified Formulation (CUF) (see, for instance, [44,45]). Within this framework, and after introducing a nondimensional reference system where $\xi=\frac{2 x}{a}, \eta=\frac{2 y}{b}$ and $\zeta=\frac{2 z}{h}$, the displacement field components are axiomatically expressed as the product of thickness functions and generalized displacement components:

$u_{r}^{k}(\xi, \eta, \zeta)=F_{\alpha_{u_{r}}}(\zeta) u_{r \alpha_{u r}}^{k}(\xi, \eta) \quad$ with $\quad r=x, y, x \quad$ and $\quad \alpha_{u_{r}}=0,1, \ldots, N_{r}$

where the sum is implied with respect to the repeated index $\alpha_{u_{r}}$ and the thickness functions $F_{\alpha_{u r}}$ are taken as proper combinations of Legendre polynomials [36,37,41,44-46]. According to Eq. (4), each displacement component $u_{r}$ can be expanded independently from the other ones. Both equivalent single-layer (ED) and layerwise (LD) theories can be retrieved in the context of the same framework, depending on the assembly procedure of the governing equations. Each theory is identified with an acronym denoting the typology of the kinematic description and the order of expansion of each displacement component. For example, the theory $\mathrm{ED}_{332}$ stands for an equivalent single-layer theory where the global displacements $u_{x}$ and $u_{y}$ are expanded up to third order whilst $u_{z}$ is described by a second-order expansion. Likewise, the theory $\mathrm{LD}_{444}$ identifies a layerwise kinematics where the local displacement components $u_{x}^{k}, u_{y}^{k}$ and $u_{z}^{k}$ in each layer $k$ of the laminate are expanded up to fourth order. Note also that $\mathrm{ED}_{110}$ corresponds to the assumed displacement field of the well-known first-order shear deformation theory, which will be denoted in the following by the common acronym FSDT.

\subsection{Ritz approximation}

The generalized displacement components $u_{r u_{u r}}^{k}$ of Eq. (4) are functions of the in-plane coordinates $\xi$ and $\eta$, as the dependency on the thickness-wise coordinate is condensed in the thickness functions $F_{\alpha_{u r}}$. Referring to the Ritz method, they are approximated by means of global admissible functions, whose general expression is given by:

$u_{r \alpha_{u r}}^{k}(\xi, \eta)=N_{u_{r} i}(\xi, \eta) u_{r \alpha_{r} i}^{k} \quad$ with $\quad i=1, \ldots, M=R \times S$

where $N_{u_{r} i}$ is the generic ith function, $R$ and $S$ denote the number of terms of the approximation along the $x$ and $y$ direction, respectively. For a rectangular domain, the trial functions are represented by assuming separation of variables as:

$N_{u_{r} i}(\xi, \eta)=\phi_{u_{r} m}(\xi) \psi_{u_{r} n}(\eta) \quad m=1, \ldots, R \quad n=1, \ldots, S$

where the functions are identified so that the relation between the indexes $m, n$ and $i$ is given by:

$i=S(m-1)+n$

The Ritz approximation of the PVD is readily obtained after substituting Eq. (6) into Eq. (3). By adopting a compact notation, where the separation between thickness and in-plane integrals is highlighted, the expressions reads:

$\delta \mathbf{u}_{r i}^{\mathrm{T}}\left(\mathbf{Z}_{(\partial) u_{r}(\partial) u_{S} R S}+\mathbf{Z}_{(\partial) u_{r}(\partial) u_{S} G}\right) \mathscr{I}_{u_{r} u_{s} i j}^{\text {defg }} \mathbf{u}_{s j}=-\delta \mathbf{u}_{r i}^{\mathrm{T}} \mathbf{Z}_{u_{r} u_{s} \rho} \mathscr{I}_{u_{r} u_{S} i j}^{\text {defg }} \ddot{\mathbf{u}}_{s j}$

where the sum is intended with respect to the repeated indexes $r, s, i$ and $j$. The matrices $\mathbf{Z}_{(\partial) u_{r}(\partial) u_{s} R S}, \mathbf{Z}_{(\partial) u_{r}(\partial) u_{s} G}$ and $\mathbf{Z}_{u_{r} u_{s} \rho}$ are achieved after integrating and assembling the thickness integrals as discussed in Ref. [38]; note that, according to the compact notation of Eq. (8), the symbol ( $\partial$ ) denotes that the terms composing $\mathbf{Z}_{(\partial) u_{r}(\partial) u_{s} R S}$ may or may not be characterized by the presence of a derivative with respect to $z$ of the thickness function. The term $\mathscr{I}_{u_{r} u_{s} i j}^{\text {defg }_{i j}}$ represents the generic ijth component of the matrix collecting the in-plane integrals of the admissible functions, which is sometimes referred to as Ritz integrals matrix. In particular, their expression is given as:

$$
\begin{aligned}
& \mathscr{I}_{u_{r} u_{s} i j}^{\text {defg }}=\left(\mathcal{I}_{u_{r} u_{s}}^{\text {defg }}\right)_{i j}=\int_{-1}^{1} \int_{-1}^{1} \frac{\partial^{d+e} N_{u_{r} i}}{\partial x^{d} \partial y^{e}} \frac{\partial f+g_{N_{u_{s} j}}}{\partial x} \mathrm{~d} \eta \mathrm{d} \xi \xi \\
& =\int_{-1}^{1} \int_{-1}^{1} N_{u_{r} i}^{(d+e)} N_{u_{S} j}^{(f+g)} \mathrm{d} \eta \mathrm{d} \xi \quad \text { with } \quad i, j=1, \ldots, R \times S \\
& (d, e, f, g=0,1)
\end{aligned}
$$

where the notation $(\mathbf{A})_{i j}$ is used to denote the component $i j$ of the generic matrix $\mathbf{A}$, while a compact notation is adopted for denoting the differentiation with respect to $\xi$ and $\eta$ as:

$\begin{array}{ll}(\bullet)^{(i)}=\frac{\partial^{i} \bullet}{\partial \xi^{i}} & (i=d, f) \\ (\bullet)^{(i)}=\frac{\partial^{i} \bullet}{\partial \eta^{i}} & (i=e, g)\end{array}$

According to Eqs. (9) and (10) and the separation of variables introduced in Eq. (6), the derivatives of the admissible functions are written as:

$N_{u_{r} i}^{(d+e)}(\xi, \eta)=\Phi_{u_{r} m}^{(d)}(\xi) \Psi_{u_{r} n}^{(e)}(\eta) \quad N_{u_{s} j}^{(f+g)}(\xi, \eta)=\Phi_{u_{s} \bar{m}}^{(f)}(\xi) \Psi_{u_{s} \bar{n}}^{(g)}(\eta)$

After substituting Eq. (11) into Eq. (9), the matrix of Ritz integrals can be re-organized as:

$\left(\mathcal{I}_{u_{r} u_{s}}^{\text {defg }}\right)_{i j}=\int_{-1}^{+1} \Phi_{u_{r} m}^{(d)} \Phi_{u_{s} \bar{m}}^{(f)} \mathrm{d} \xi \int_{-1}^{+1} \Psi_{u_{r} n}^{(d)} \Psi_{u_{s} \bar{n}}^{(f)} \mathrm{d} \eta$

From the previous definitions, the stiffness and mass matrices $\mathbf{K}$ and $\mathbf{M}$ are obtained by combining the Ritz and the thickness integrals according to the Kronecker product:

$\mathbf{K}=\sum_{r s} \mathcal{I}_{u_{r} u_{s}}^{\text {defg }} \otimes \mathbf{Z}_{(\partial) u_{r}(\partial) u_{s} R S} \quad r, s=x, y, z$

$\mathbf{M}=\sum_{r s} \mathcal{I}_{u_{r} u_{s}}^{\text {defg }} \otimes \mathbf{Z}_{u_{r} u_{s} \rho} \quad r, s=x, y, z$

$\mathbf{G}=\sum_{r s} \mathcal{I}_{u_{r} u_{s}}^{\operatorname{defg}_{s}} \otimes \mathbf{Z}_{u_{r} u_{s} G} \quad r, s=x, y, z$

The thickness integrals appearing in Eqs. (13)-(15) can be easily determined after analytically integrating the thickness functions along the normal direction. On the other hand, the evaluation of the Ritz integrals $\mathcal{I}_{u_{r} u_{S}}^{\text {defg }}$ can be a lengthy and onerous operation, thus affecting the performance of the method. For this reason, its efficient evaluation is outlined in the next paragraph.

The final form of the discrete governing equations is obtained as:

$\left(-\omega^{2} \mathbf{M}+\mathbf{K}+\lambda \mathbf{G}\right) \mathbf{u}=\mathbf{0}$

where the free vibration problem is solved by neglecting the geometric stiffness $\mathbf{G}$, whilst the buckling analysis is conducted by setting to zero the mass matrix $\mathbf{M}$. 


\subsection{Efficient computation of the Ritz integrals}

The efficient evaluation of the matrix collecting the Ritz integrals is one of the significant aspects of the present formulation. The approach discussed here is developed to guarantee improved performance, a necessary feature for dealing with highly anisotropic plates, as discussed later.

The square matrix $\mathcal{I}_{u_{r} u_{s}}^{\text {defg }}$ has dimensions $R S \times R S$. This means that, potentially, several computations need to be performed when the number of functions is not restricted to just a few terms. This difficulty is here overcome by introducing the so called integrals kernels - matrices of smaller dimensions collecting the integrals -, which are successively expanded and properly assembled to obtain Eq. (9). The efficiency of the approach is further improved with the help of the closed-form integration of the integral kernels themselves. This operation, which is performed for all the admissible functions discussed in the present work, is carried out by symbolic computations using Mathematica.

The two kernels, which are the integrals of the trial functions associated with the displacement components $u_{r}$ and $u_{s}$ along the directions $\xi$ and $\eta$, are indicated as:

$$
\begin{aligned}
& \left(\mathbf{I}_{u_{r} u_{s}}^{d f}\right)_{m p}=\int_{-1}^{+1} \phi_{u_{r} m}^{(d)} \phi_{u_{s} p}^{(f)} \mathrm{d} \xi \quad \text { with } \quad m, p=1, \ldots, R \\
& \left(\mathbf{J}_{u_{r} u_{S}}^{e g}\right)_{n q}=\int_{-1}^{+1} \psi_{u_{r} n}^{(e)} \psi_{u_{s} q}^{(g)} \mathrm{d} \xi \quad \text { with } \quad n, q=1, \ldots, S
\end{aligned}
$$

It can be noted that the two matrices of Eq. (17) are characterized by dimensions $R \times R$ and $S \times S$, respectively. Their evaluation, which suffices for the successive construction of the final matrix of Ritz integrals of Eq. (9), can thus be performed by computing just $(R \times R)+(S \times S)$ integrals. The kernels of Eq. (17) are computed for a sufficiently large number of functions (up to 250) just once, and are stored in a binary file. At every run, the kernels are simply loaded from the binary file, and used to construct the matrix of Ritz integrals with a minimum computational effort. By using the Kronecker product, the kernel of the integrals $\left.\right|_{u_{r} u_{s}} ^{e g}$ can be expanded as:

$$
\begin{aligned}
\mathbf{I}_{u_{r} u_{S}}^{d f} & =\int_{-1}^{+1} \phi_{u_{r} m}^{(d)} \phi_{u_{S} \bar{m}}^{(f)} \mathrm{d} \xi \quad \text { with } \quad m=m(i), \text { and } \bar{m}=\bar{m}(i) \\
& =\mathbf{I}_{u_{r} u_{S}}^{d f} \otimes \mathbf{I}_{S S} \quad \text { and } \quad i=1, \ldots, R \times S
\end{aligned}
$$

where $\mathbf{I}_{S S}$ is the identity matrix of dimension $S \times S$, and the resulting matrix $\mathbf{I}_{u_{r} u_{S}}^{d f}$ has dimension $R S \times R S$.

Similarly, the expansion of the kernels $\mathbf{J}_{u_{r} u_{s}}^{e g}$ is obtained as:

$$
\begin{aligned}
\mathbf{J}_{u_{r} u_{S}}^{e g} & =\int_{-1}^{+1} \psi_{u_{r} n}^{(e)} \psi_{u_{s} \bar{n}}^{(g)} \mathrm{d} \eta \quad \text { with } \quad n=n(j), \text { and } \bar{n}=\bar{n}(j) \\
& =\mathbf{I}_{R R} \otimes \mathbf{J}_{u_{r} u_{s}}^{e g} \quad \text { and } \quad j=1, \ldots, R \times S
\end{aligned}
$$

where $\mathbf{I}_{R R}$ is the identity matrix of dimension $R \times R$, and the expanded matrix of integrals $\mathbf{J}_{u_{r} u_{S}}^{e g}$ has dimension $R S \times R S$.

Recalling now Eq. (12), it is possible to express the matrix of the Ritz integrals as the Hadamard elementwise product between the expanded kernels of the integrals along the directions $\xi$ and $\eta$ (Eqs. (18) and (19)):

$\mathcal{I}_{u_{r} u_{s}}^{\text {defg }}=\mathbf{I}_{u_{r} u_{s}}^{d f} \circ \mathbf{J}_{u_{r} u_{s}}^{e g}$

For clarity, a graphical description of the procedure for evaluating the Ritz integrals is provided in Fig. 2. Without loss of generality, it is considered the case where an expansion $3 \times 2$ is adopted. Highlighted is the way the kernels are initially expanded (Eqs. (18) and (19)) and the resulting matrices are successively multiplied through the Hadamard product (Eq. (20)).

\subsection{Admissible functions}

As outlined in the Introduction, different sets of functions can be used for approximating the displacement field and this choice plays a crucial role in the development of a Ritz-based procedure. Indeed, several properties are affected by the functions adopted, including the convergence, efficiency, accuracy and numerical stability of the method. In the present work, two classes of orthogonal polynomials are considered, namely Legendre and Chebyshev polynomials, and two sets of trigonometric functions. The first is referred to as Navier-type expansion, consisting in the set of sines and cosines descending from the classical Navier-solution of those problem for which an exact solution can be sought. The second trigonometric set is denoted as extended trigonometric, and represents an enrichment of the Navier-type basis resulting from the introduction of linear polynomial contributions. The implementation of different sets of functions allows one to compare the quality of the various choices, and demonstrates the inadequacy of improperly adopting the Navier-type expansion, unless special conditions are met.

\subsubsection{Orthogonal polynomials}

In the case of orthogonal polynomials, the one-dimensional functions $\phi_{u_{r} m}(\xi)$ and $\psi_{u_{r} n}(\eta)$ are expressed by taking the product with boundary functions, properly selected to impose the fulfillment of the essential boundary conditions:

$\phi_{u_{r} m}(\xi)=f_{u_{r}}(\xi) p_{m}(\xi)$

$\psi_{u_{r} n}(\eta)=g_{u_{r}}(\eta) p_{n}(\eta)$

where $f_{u_{r}}(\xi)$ and $g_{u_{r}}(\eta)$ are the boundary functions, defined as:

$f_{u_{r}}(\xi)=(1+\xi)^{e_{1 r}}(1-\xi)^{e_{2 r}}$

$g_{u_{r}}(\eta)=(1+\eta)^{e_{1 r}(1-\eta)^{e_{2 r}}}$

The coefficients $e_{1 r}$ and $e_{2 r}$ can be either 0 or 1 , and are chosen depending on the boundary conditions [36]. In the particular case of CLT, the coefficients can be 0,1 or 2 for free, pinned and clamped conditions, respectively.

Although any class of orthogonal polynomials could be implemented within the present framework, the functions $p_{m}$ and $p_{n}$ are here expressed using Chebyshev or Legendre polynomials. In the first case, the polynomials are expressed as:

$p_{l}(\chi)=\cos [(l-1) \arccos (\chi)] \quad$ with $l=m, n$

while for the case of Legendre polynomials the expression is defined according to the recursion formula:

$p_{0}=1 ; \quad p_{1}=\chi ; \quad p_{l+1}=\frac{(2 l+1) \chi p_{l}-l p_{l-1}}{l+1} \quad$ with $l=m, n$

\subsubsection{Trigonometric functions}

The second class of functions discussed here is given by trigonometric functions. Their use is mainly restricted to the case of simplysupported boundary conditions, and have been widely adopted in the literature [30-34]. One of the main reasons motivating their popularity is the simplicity of the resulting in-plane integrals. In addition, the resulting matrices do not suffer from ill-conditioning problems, as it may happen for some class of polynomial expansions.

In the Navier-type expansions, the three components of the displacement field are expressed as:

$N_{u_{x} i}(\xi, \eta)=\cos \frac{m \pi}{2}(\xi+1) \sin \frac{n \pi}{2}(\eta+1)$
$N_{u_{y} i}(\xi, \eta)=\sin \frac{m \pi}{2}(\xi+1) \cos \frac{n \pi}{2}(\eta+1)$
$N_{u_{z} i}(\xi, \eta)=\sin \frac{m \pi}{2}(\xi+1) \sin \frac{n \pi}{2}(\eta+1)$

where the relation between the indexes $i, m$ and $n$ is given by Eq. (7). The expressions of Eq. (25) are suitable for modeling simply-supported boundary conditions, meaning that the out-of-plane displacement and the component tangential to the panel edges are assumed to be null along the plate boundaries.

Similarly, the extended trigonometric representation is achieved by adding linear polynomial contributions to Eq. (25) as: 


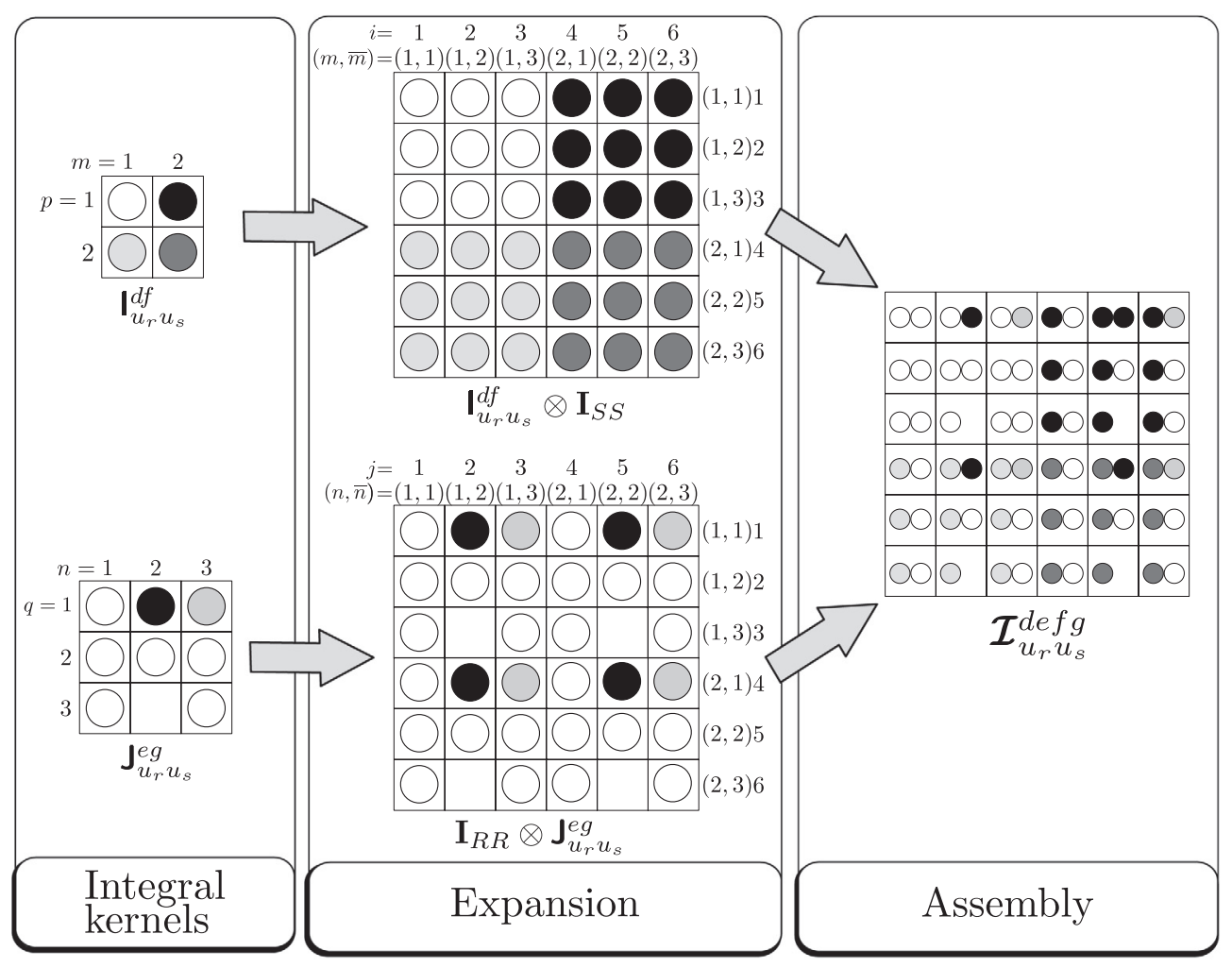

Fig. 2. Construction of the Ritz integrals matrix $(R=2$ and $S=3)$ : evaluation of the Ritz kernels, expansion and final assembly.

$$
\begin{array}{ll}
N_{u_{x} 0}(\xi, \eta)=\frac{1}{2}(\xi+1) & N_{u_{x} i}(\xi, \eta)=\cos \frac{m \pi}{2}(\xi+1) \sin \frac{n \pi}{2}(\eta+1) \\
N_{u_{y} 0}(\xi, \eta)=\frac{1}{2}(\eta+1) & N_{u_{y} i}(\xi, \eta)=\sin \frac{m \pi}{2}(\xi+1) \cos \frac{n \pi}{2}(\eta+1) \\
N_{u_{z} i}(\xi, \eta)=\sin \frac{m \pi}{2}(\xi+1) \sin \frac{n \pi}{2}(\eta+1)
\end{array}
$$

Combination of trigonometric and polynomial terms have been successfully used in the literature, for example see Monterrubio and Ilanko [47]. The enrichment of the basis by means of linear polynomial terms is essential for guaranteeing the completeness of the basis in the energy norm [48], as shown in the next section. The polynomial contributions are quite often neglected, and the basis is formally identical to the displacement field description adopted in the context of Naviertype solutions. As such, it can be successfully applied to those cases where an exact Navier-type solution is available. In all the other cases, convergence to the exact solution cannot be achieved if Eq. (25) is adopted.

It is finally remarked that classical Fourier analysis could be used for improving the convergence of the results [49-53]. However, this is not the approach adopted here and the derivatives of the displacement field are assumed to be term-by-term differentiable.

\section{Results}

This section aims at illustrating and discussing some results on free vibration and buckling of highly anisotropic rectangular plates obtained from Ritz models based on CLT and the variable-kinematic formulation. All the plates analyzed throughout the section are made of the material P100/AS3501, taken from Refs. [54,29], whose elastic properties are the following: $E_{1}=369 \mathrm{GPa}, E_{2}=5030 \mathrm{MPa}, G_{12}=5240 \mathrm{MPa}$, and $\nu_{12}=0.31$. In addition, it is assumed that $G_{13}=G_{23}=G_{12}$ and $v_{13}=v_{23}=v_{12}$, and the density $\rho$ is taken equal to $1500 \mathrm{~kg} / \mathrm{m}^{3}$. As seen, this material is characterized by a high orthotropy ratio $E_{1} / E_{2}>70$, whose effect is to exacerbate the amount of anisotropy of all the lay-ups considered, and highlight any potential convergence issue of the discretization method. All the results are expressed in terms of the nondimensional quantities $\bar{\omega}_{i}$ and $\bar{N}_{x}$ for the free vibration and the buckling force per unit length, respectively, as:

$\bar{\omega}_{i}=\omega_{i} \frac{a^{2}}{h} \sqrt{\frac{\rho}{E_{2}}} \quad \bar{N}_{x}=N_{x} \frac{a^{2}}{E_{2} h^{3}}$

where $a$ is the plate longitudinal dimension, $h$ is the thickness, $\rho$ the density and $E_{2}$ the transverse Young's modulus; $N_{x}$ denotes the force per unit length directed parallel to the $x$ axis, and applied at the edges at $x=$ const.

This section is organized as follows: thin plates are analyzed first in Section 3.1 by considering orthogonal polynomials and extended trigonometric functions. The effects due to different kinds of elastic couplings, material anisotropy as well as the role of boundary conditions are assessed. Novel benchmark results are also obtained by exploiting the efficiency of the present implementation for highly anisotropic plates, where a huge number of terms in the Ritz series is required to achieve a satisfactory level of accuracy of the solution. The investigation is then extended to the case of thick plates in Section 3.2. The requirements on the completeness of the admissible set are discussed in Section 3.3, and exemplary results are provided for highlighting potential errors associated with an improper use of a Navier-type expansion. Finally, the computational efficiency of the various sets of functions is discussed in Section 3.4, and the superiority of Legendre polynomials is illustrated.

\subsection{Thin plates}

\subsubsection{Anisotropic laminates with different elastic couplings}

A set of four thin-plate configurations is initially analyzed for highlighting the effects of different kinds of elastic couplings on the free vibration response. The results are presented in the context of CLT, which is commonly adopted for thin plate analysis and for validating novel numerical techniques (see, for instance, Refs. [55-57]). Note that rotatory inertia is neglected from the following computations. Square plates are considered, and characterized by four distinct lay-ups, 
hereinafter denoted as:

- Lay-up L1: [45/45]

- Lay-up L2: [0/90]

- Lay-up L3: [45/-45]

- Lay-up L4: [0/45]

It is worth noting that the nature of the elastic couplings exhibited by the above lay-ups in terms of the familiar A,B and D matrices of CLTbased models (see, e.g. [58-60]) is inherently different. The first lay-up is symmetric and characterized by bending/twisting coupling; the second one is cross-ply, non-symmetric with coupled in-plane and outof-plane behavior due to not vanishing terms $B_{11}$ and $B_{22}=-B_{11}$; L3 displays another sort of coupling between in-plane and out-plane response through the terms $B_{16}$ and $B_{26}$; finally, L4 is also a non-symmetric configuration and exhibits a fully populated ABD constitutive relation.

Simply-supported boundary conditions are assumed along the four edges. This means that, in addition to the out-of-plane displacement, the tangential in-plane component is prevented from motion, while the normal one is free. In this first assessment, the admissible functions are Legendre polynomials - it is here anticipated that identical results are obtained using Chebyshev polynomials - and extended trigonometric functions. The first three nondimensional frequencies $\bar{\omega}_{i}$ are summarized in Table 1 for an increasing number $R=S$ of functions. It is noted at the outset that computations using extended trigonometric functions are shown for a limited number of 50 functions along each direction, due to their computational inefficiency, as addressed in more detail later in Section 3.4. On the contrary, Legendre polynomials can be easily employed to consider higher number of terms in the Ritz series, and frequency parameters are presented for $R$ up to 100 .

The comparison is presented against refined finite element analysis, obtained using Abaqus S4 and S4R four-node shell elements. A mesh size of $400 \times 400$ elements is defined on the basis of a preliminary study aimed at guaranteeing convergence of the finite element solution up to the second digit. As far as S4R and S4 are general purpose elements, the numerical models are realized by considering an artificially high ratio $a / h=10000$, in order to keep at minimum shear deformation effects. Note that the sole scope of adopting such a high value is to perform a fair comparison with CLT-based results obtained using Ritz. For layup L2, the exact Navier-type solution is available, which is then taken as a reference instead of the finite element one.

The results of Table 1 illustrate the excellent level of agreement between reference results and those obtained with the Ritz discretization. The percent differences, reported in the parenthesis, provide a clear insight into the convergence of the solution and the differences arising from the adoption of trigonometric and Legendre trial functions. It can be observed that orthogonal polynomials lead to results which are almost identical to the reference solutions, provided the number of functions is sufficiently high.

The most critical case is given by lay-up L1, which is the one characterized by the slowest convergence rate. For this case, the results are still slightly different from the reference ones, even when 100 functions are used along both the directions. This behaviour is motivated by the strong bending/twisting coupling, which makes the solution unavailable by separation of variables, as demonstrated by Wang [61].

From Table 1, it is also clearly visible that convergence is much faster for Legendre polynomials than trigonometric functions. For layup L1, errors higher than $6 \%$ are obtained on the fundamental frequency of the plate even for trigonometric expansions of 50 terms. The only exception is given by lay-up L2, where the present solution shows no difference with the exact solution. Indeed, L2 is a cross-ply configuration, for which the exact solution exists in trigonometric form. For this special case, the exact solution is thus exactly matched by the trigonometric set assumed in the Ritz method.

It is worth noting that the Ritz results of Table 1 are upper bound predictions. This is not the case for the Abaqus S4R results, which are based upon reduced integration, thus convergence from above cannot be guaranteed. When comparing Ritz solutions with Abaqus S4 results, it is observed that frequency values obtained with S4 elements are higher than predictions computed by the Ritz method using Legendre

Table 1

Nondimensional frequencies $\bar{\omega}_{i}$ for SSSS thin square plates, using CLT and $R \times R$ functions. (Note: ${ }^{\text {a }}$ used as reference for percent difference evaluation).

\begin{tabular}{|c|c|c|c|c|c|c|c|}
\hline \multirow[b]{2}{*}{ Layup } & \multirow[b]{2}{*}{$\mathrm{R}$} & \multicolumn{2}{|c|}{$\bar{\omega}_{1}$} & \multicolumn{2}{|c|}{$\bar{\omega}_{2}$} & \multicolumn{2}{|c|}{$\bar{\omega}_{3}$} \\
\hline & & Legendre & Extended trig. & Legendre & Extended trig. & Legendre & Extended trig. \\
\hline \multirow[t]{6}{*}{ L1 } & 5 & $23.7290(7.88)$ & $26.6999(21.39)$ & $37.2524(2.75)$ & $42.1941(16.38)$ & $61.1104(14.32)$ & 62.3625 (16.67) \\
\hline & 10 & $22.8746(4.00)$ & $25.4317(15.62)$ & $36.2590(0.01)$ & 39.1541 (7.99) & $53.6133(0.30)$ & $58.1908(8.86)$ \\
\hline & 20 & $22.4097(1.88)$ & $24.4310(11.07)$ & $36.2547(0.00)$ & $37.8074(4.28)$ & $53.5257(0.14)$ & $56.1407(5.03)$ \\
\hline & 50 & $22.1228(0.58)$ & $23.4815(6.76)$ & $36.2546(0.00)$ & 36.9171 (1.82) & $53.4731(0.04)$ & $54.7166(2.36)$ \\
\hline & 100 & $22.0258(0.14)$ & / & $36.2546(0.00)$ & / & $53.4556(0.00)$ & / \\
\hline & S4R-S4 & $21.9952^{\mathrm{a}}-22.2083$ & & $36.2562^{\mathrm{a}}-36.2566$ & & $53.4535^{\mathrm{a}}-53.4928$ & \\
\hline \multirow[t]{6}{*}{ L2 } & 5 & $14.5082(0.02)$ & $14.5056(0.00)$ & $40.5196(0.37)$ & $40.3701(0.00)$ & $40.5196(0.37)$ & $40.3701(0.00)$ \\
\hline & 10 & $14.5056(0.00)$ & $14.5056(0.00)$ & $40.3701(0.00)$ & $40.3701(0.00)$ & $40.3701(0.00)$ & $40.3701(0.00)$ \\
\hline & 20 & $14.5056(0.00)$ & $14.5056(0.00)$ & $40.3701(0.00)$ & $40.3701(0.00)$ & $40.3701(0.00)$ & $40.3701(0.00)$ \\
\hline & 50 & $14.5056(0.00)$ & $14.5056(0.00)$ & $40.3701(0.00)$ & $40.3701(0.00)$ & $40.3701(0.00)$ & $40.3701(0.00)$ \\
\hline & 100 & $14.5056(0.00)$ & / & $40.3701(0.00)$ & / & $40.3701(0.00)$ & 1 \\
\hline & Exact & $14.5056^{\mathrm{a}}$ & & $40.3701^{\mathrm{a}}$ & & $40.3701^{\mathrm{a}}$ & \\
\hline \multirow[t]{6}{*}{ L3 } & 5 & $23.8777(0.15)$ & $26.0692(9.35)$ & $45.3719(1.81)$ & $49.4182(10.8)$ & 45.3719 (1.81) & 49.4379 (10.93) \\
\hline & 10 & $23.8452(0.02)$ & $25.0085(4.90)$ & $44.6529(0.19)$ & $47.0179(5.50)$ & $44.6529(0.19)$ & $47.0189(5.50)$ \\
\hline & 20 & $23.8415(0.00)$ & $24.4367(2.50)$ & $44.5865(0.04)$ & $45.8972(2.99)$ & $44.5865(0.04)$ & 45.8973 (2.99) \\
\hline & 50 & $23.8407(0.00)$ & $24.0836(1.02)$ & $44.5692(0.01)$ & 45.1430 (1.29) & $44.5692(0.01)$ & 45.1431 (1.29) \\
\hline & 100 & $23.8406(0.00)$ & / & $44.5669(0.00)$ & / & $44.5669(0.00)$ & / \\
\hline & S4R-S4 & $23.8409^{\mathrm{a}}-23.8409$ & & $44.5668^{\mathrm{a}}-44.5729$ & & $44.5668^{\mathrm{a}}-44.5729$ & \\
\hline \multirow[t]{6}{*}{ L4 } & 5 & $17.3976(0.48)$ & $17.9870(3.88)$ & $31.7112(1.66)$ & 33.7065 (8.06) & $52.3862(1.85)$ & 53.3507 (3.73) \\
\hline & 10 & $17.3405(0.15)$ & 17.6933 (2.19) & $31.3584(0.53)$ & $32.5094(4.22)$ & $51.6209(0.37)$ & $52.5177(2.11)$ \\
\hline & 20 & $17.3235(0.05)$ & $17.5289(1.24)$ & $31.2574(0.20)$ & 31.9391 (2.39) & $51.5048(0.14)$ & $52.0596(1.22)$ \\
\hline & 50 & $17.3168(0.01)$ & $17.4107(0.56)$ & $31.2094(0.05)$ & 31.5511 (1.15) & $51.4497(0.03)$ & $51.7468(0.61)$ \\
\hline & 100 & $17.3152(0.00)$ & / & $31.1973(0.01)$ & / & $51.4358(0.01)$ & / \\
\hline & S4R-S4 & $17.3145^{\mathrm{a}}-17.3188$ & & $31.1935^{\mathrm{a}}-31.2202$ & & $51.4323^{\mathrm{a}}-51.4627$ & \\
\hline
\end{tabular}


Table 2

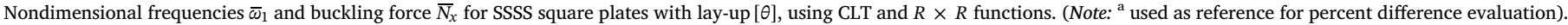

\begin{tabular}{|c|c|c|c|c|c|c|c|}
\hline & & \multicolumn{2}{|c|}{$\theta=15$} & \multicolumn{2}{|c|}{$\theta=30$} & \multicolumn{2}{|c|}{$\theta=45$} \\
\hline & & Legendre & Extended trig. & Legendre & Extended trig. & Legendre & Extended trig. \\
\hline \multirow{7}{*}{$\bar{\omega}_{1}$} & 5 & $24.6447(0.45)$ & $25.2133(2.76)$ & $23.5729(3.83)$ & 25.7182 (13.27) & $23.7290(7.88)$ & 26.6999 (21.39) \\
\hline & 10 & $24.5725(0.15)$ & $24.9119(1.54)$ & $23.1193(1.83)$ & $24.7275(8.91)$ & $22.8746(4.00)$ & 25.4317 (15.62) \\
\hline & 20 & $24.5464(0.05)$ & $24.7450(0.86)$ & $22.8890(0.81)$ & $24.0354(5.86)$ & $22.4097(1.88)$ & 24.4310 (11.07) \\
\hline & 40 & $24.5378(0.01)$ & $24.6516(0.48)$ & $22.7773(0.32)$ & $23.5741(3.83)$ & $22.1711(0.80)$ & $23.6767(7.64)$ \\
\hline & 50 & $24.5366(0.01)$ & $24.6313(0.39)$ & $22.7559(0.23)$ & $23.4614(3.33)$ & $22.1228(0.58)$ & $23.4815(6.76)$ \\
\hline & 100 & $24.5349(0.00)$ & / & $22.7151(0.05)$ & / & $22.0258(0.14)$ & / \\
\hline & Abaqus S4R-S4 & $24.5350^{\mathrm{a}}-24.5399$ & & $22.7043^{\mathrm{a}}-22.7968$ & & $21.9952^{\mathrm{a}}-22.2083$ & \\
\hline \multirow{7}{*}{$\overline{N_{x}}$} & 5 & $57.5058(0.97)$ & $60.3686(6.00)$ & 43.4565 (11.79) & $52.0261(33.84)$ & $38.6798(27.53)$ & 44.9187 (48.10) \\
\hline & 10 & $57.1270(0.30)$ & $58.8198(3.28)$ & $40.2498(3.54)$ & $46.7365(20.23)$ & $32.7145(7.86)$ & 38.8464 (28.07) \\
\hline & 20 & $57.0064(0.09)$ & $57.9766(1.80)$ & 39.4905 (1.59) & $43.8220(12.73)$ & $31.4656(3.74)$ & $36.2285(19.44)$ \\
\hline & 40 & $56.9669(0.02)$ & $57.5164(0.99)$ & $39.1168(0.63)$ & $42.0201(8.10)$ & 30.8137 (1.59) & 34.7958 (14.72) \\
\hline & 50 & $56.9614(0.01)$ & $57.4174(0.81)$ & $39.0449(0.44)$ & $41.5943(7.00)$ & 30.6813 (1.15) & 34.4867 (13.70) \\
\hline & 100 & $56.9534(0.00)$ & / & $38.9076(0.09)$ & / & 30.4147 & / \\
\hline & Abaqus S4R-S4 & $56.9535^{\mathrm{a}}-56.9753$ & & $38.8728^{\mathrm{a}}-39.1825$ & & $30.3310^{\mathrm{a}}-30.9177$ & \\
\hline
\end{tabular}

polynomials. Recalling that FEM results are here obtained with highly refined meshes, it can be noted the better accuracy-to-degrees-offreedom ratio offered by the Ritz approach. With this regard, the total number of Ritz degrees of freedom, for $R$ equal to 100 , is 10000 , two orders of magnitude less than Abaqus models.

Finally, it is observed that, for the examples here proposed, the elastic coupling responsible for the most detrimental effects on the convergence is the bending/twisting one. Membrane-bending/twisting coupled behaviour, as those characterizing the constitutive law of layups L1, L3 and L4, have a minor impact on the convergence of the solutions.

\subsubsection{Effect of flxural anisotropy}

Given the effects of bending/twisting coupling on the convergence of the method, another example is considered in Table 2 involving a thin plate made of one single ply only with fibers oriented at $\theta$ degrees. Results with three different angles $\theta=15,30,45$ are reported in order to highlight how the results are affected by increasing amount of flexural anisotropy. The values in Table 2 refer to the first natural frequency and the nondimensional buckling force per unit length according to Eq. (27). The comparison is presented against refined Abaqus results obtained with S4R and S4 elements.

The results clearly show that the convergence becomes markedly slower as the flexural anisotropy, which is maximum when $\theta$ is equal to 45 , is increased. This conclusion holds both for vibration and buckling analysis. With this regard, increasing values of flexural anisotropy render trigonometric functions more and more inadequate. The errors with respect to finite element results for the configuration with $\theta$ equal to 15 are well below $1 \%$, while they are more than $3 \%$ and $6 \%$ when $\theta$ is equal to 30 and 45 .

It is worth noting that the errors achieved for the first natural frequency are, in general, smaller than those relative to the buckling force, as also observed by Stone and Chandler [24]. The main reason for this discrepancy is given by the relation between the result and the eigenvalue of the problem, and not by the kind of problem itself. More specifically, the natural frequency depends on the square root of the problem's eigenvalue, while the buckling force depends linearly on the eigenvalue itself. It can be shown that $e_{\omega} \approx \sqrt{e_{\text {buckle }}+1}-1$, where $e_{\omega}$ and $e_{\text {buckle }}$ are the ratios between the approximate and the exact solutions associated with the frequency and buckling force prediction, respectively.

For the sake of completeness, the plot in Fig. 3 illustrates the results obtained for various angles $\theta$ ranging from 0 to 90 degrees by using Legendre and trigonometric functions in the Ritz method, as compared to Abaqus S4R results.

\subsubsection{Effect of boundary conditions}

The effect of anisotropy cannot be analyzed without establishing a link with the boundary conditions. Indeed, the solution for plates characterized by the same lay-up can exhibit different convergence behaviour on the basis of the constraints specified at the boundaries. This effect is assessed in Table 3, both for the free vibration and buckling response of the same plate of Example 2 made of one single ply oriented at 45 degrees. According to the convention adopted, the first and the third edges are those at $\xi=-1$ and $\xi=+1$, while the second and the fourth ones are those at $\eta=-1$ and $\eta=+1$, respectively.

The results are presented by using a relatively low number of Legendre polynomials in the Ritz series, $R=S=20$, and by exploiting the efficiency of the implementation proposed in Section 2.3 to derive highly refined results with $100 \times 100$ functions.

As seen from Table 3 , the presence of natural boundary conditions is responsible for increased difficulties in the convergence of the solution and higher errors for a given number of admissible functions. Indeed, all of the conditions characterized by combinations of free and simplysupported conditions are the most challenging ones. This is mainly due to the difficulties of the assumed approximation, obtained by separation of variables, to approximate the vanishing moment condition along the edges. On the contrary, it can be seen that clamped conditions, which are of essential type, tend to facilitate the convergence of the solution. Whenever two or more clamped edges are present, the use of $20 \times 20$ functions is generally sufficient to obtain accurate results. In this sense, the fully clamped plate represents the most favorable case, and the use of 20 functions guarantees convergence up to the fourth digit.

The role played by the material anisotropy is assessed in Table 4, where the fundamental frequency parameter and the compressive buckling force are reported by comparing Abaqus results and Ritz solutions computed with $R=S=50$. All of the elastic constants are kept unchanged expect for $E_{1}$, which is varied to obtain different values of orthotropy ratios. As expected, the errors tend to get smaller as the ratio $E_{1} / E_{2}$ is reduced, irrespective of the constraints along the plate boundary. Indeed, smaller values of the orthotropy ratio are associated with smaller degrees of flexural anisotropy. The drop of the errors when the ratio is reduced from the original value of 73.36 to 20 is noticeable. It is worth noting that the materials commonly used nowadays in advanced composite structures are rarely associated with orthotropy ratios higher than 30-40. However, the reference case with $E_{1} / E_{2}=73.36$ is selected to exaggerate the effects of elastic couplings and show the capabilities of the method to properly handle configurations involving extreme levels of anisotropy.

\subsubsection{Benchmark results for highly anisotropic plates}

The numerical efficiency of the proposed Ritz implementation is 


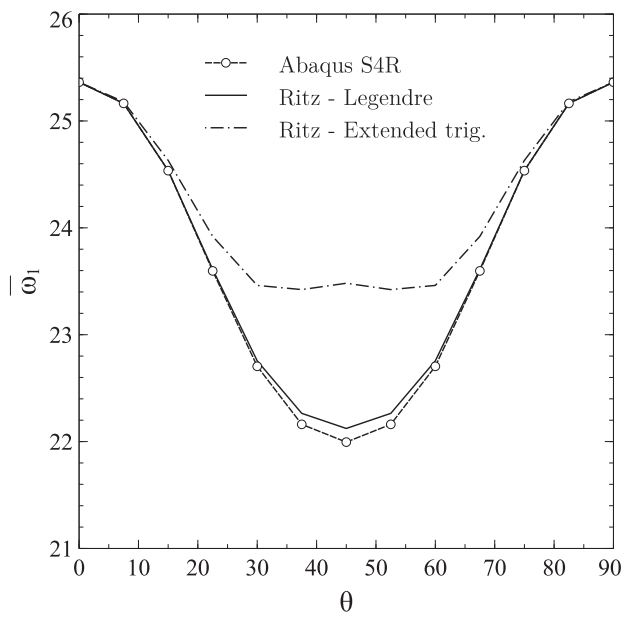

(a)

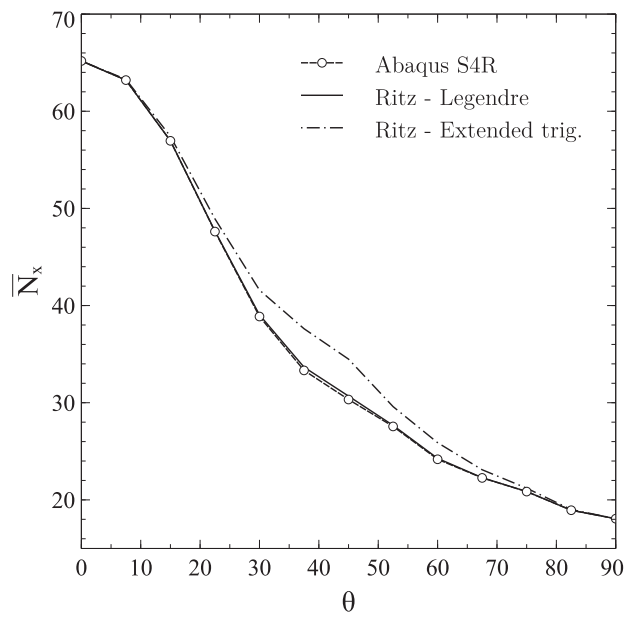

(b)

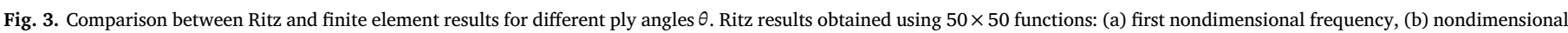
buckling force.

here exploited to obtain additional solutions for a square plate with one ply oriented at $\theta$. The following results could be used as a valuable reference for future benchmarking of novel numerical methods devoted to the analysis of anisotropic plates, where no exact solutions are available. On the basis of the results discussed in previous examples, simply-supported boundary conditions are assumed, as they constitute a challenging set of conditions for the convergence and accuracy of the method in the presence of high flexural anisotropy. Highly refined upper bound predictions are reported in Table 5 for different orthotropy ratios using up to $250 \times 250$ Legendre functions. To the best of the author's knowledge, such a level of refinement is reported for the first time in the literature. Indeed, only few dozens of admissible functions along each direction have been typically adopted in previous application of the Ritz method to anisotropic laminates. As shown before, a small number of terms in the Ritz approximation is inadequate for guaranteeing proper accuracy for problems characterized by extreme anisotropy. On the contrary, the results of Table 5 with $R=S=250$ can be considered as very accurate upper bound solutions, which may prove particularly useful as no exact results can be derived for the test cases under investigation.

\subsection{Thick plates}

This second part is devoted to the analysis of moderately thick and thick plates, with the aim of investigating how the effects of extreme anisotropy on the numerical behavior of the Ritz solution are influenced by the plate thickness ratio. The efficiency of the present implementation allows to manage Ritz approximations with a huge number of admissible functions, thus leading to vibration and buckling results for highly anisotropic plates with a degree of refinement not available in previous research efforts.

The first set of results considers square plates characterized by width-to-thickness ratios $a / h$ ranging from 5 to 100 . Two distinct configurations, characterized by elastic couplings of different nature, are analyzed. The first configuration is a symmetric lay-up with three plies at $[45 /-45 / 45]$, while the second is a non-symmetric configuration with two plies at $[ \pm 45]$.

The first fundamental frequency of each case is reported in Table 6,

Table 3

Effect of boundary conditions on the nondimensional frequencies $\bar{\omega}_{1}$ and buckling force $\bar{N}_{x}$ of thin square plates, using CLT.

\begin{tabular}{|c|c|c|c|c|c|c|c|c|c|c|}
\hline \multirow[b]{3}{*}{ CCCC } & \multicolumn{5}{|c|}{$\bar{\omega}_{1}$} & \multicolumn{5}{|c|}{$\bar{N}_{x}$} \\
\hline & \multicolumn{2}{|c|}{$20 \times 20$} & \multicolumn{2}{|c|}{$100 \times 100$} & \multirow{2}{*}{$\begin{array}{l}\text { Abaqus S4R } \\
40.7744\end{array}$} & \multicolumn{2}{|c|}{$20 \times 20$} & \multicolumn{2}{|c|}{$100 \times 100$} & \multirow{2}{*}{$\begin{array}{l}\text { Abaqus S4R } \\
55.3521\end{array}$} \\
\hline & 40.7737 & $(0.00)$ & 40.7733 & $(0.00)$ & & 55.3424 & $(-0.02)$ & 55.3414 & $(-0.02)$ & \\
\hline CCCF & 20.2789 & $(0.11)$ & 20.2587 & $(0.01)$ & 20.2575 & 21.6492 & $(0.10)$ & 21.6263 & $(0.00)$ & 21.6266 \\
\hline CCCS & 35.5083 & $(0.07)$ & 35.4818 & $(0.00)$ & 35.4826 & 46.8415 & $(0.08)$ & 46.7962 & $(-0.02)$ & 46.8034 \\
\hline CCFF & 8.4186 & $(0.00)$ & 8.4186 & $(0.00)$ & 8.4190 & 11.3127 & $(-0.01)$ & 11.3127 & $(-0.01)$ & 11.3133 \\
\hline CCSF & 18.5710 & $(0.12)$ & 18.5478 & $(0.00)$ & 18.5485 & 20.2404 & $(0.17)$ & 20.2049 & $(-0.01)$ & 20.2064 \\
\hline CCSS & 30.8442 & $(0.86)$ & 30.6018 & $(0.06)$ & 30.5823 & 42.8148 & $(1.62)$ & 42.1822 & $(0.11)$ & 42.1342 \\
\hline CFCF & 19.4496 & $(0.15)$ & 19.4214 & $(0.01)$ & 19.4203 & 20.5305 & $(0.06)$ & 20.5162 & $(-0.01)$ & 20.5175 \\
\hline CFFF & 2.1652 & $(0.23)$ & 2.1597 & $(-0.03)$ & 2.1603 & 0.7140 & $(0.29)$ & 0.7115 & $(-0.06)$ & 0.7119 \\
\hline CFSF & 9.7317 & $(0.13)$ & 9.7199 & $(0.01)$ & 9.7193 & 6.8058 & $(0.13)$ & 6.7970 & $(0.00)$ & 6.7968 \\
\hline CSCF & 20.1439 & $(0.12)$ & 20.1213 & $(0.01)$ & 20.1202 & 21.4780 & $(0.13)$ & 21.4503 & $(0.00)$ & 21.4507 \\
\hline CSFF & 4.6943 & $(0.12)$ & 4.6887 & $(0.00)$ & 4.6889 & 3.5002 & $(0.12)$ & 3.4956 & $(-0.01)$ & 3.4959 \\
\hline CSSF & 18.0708 & $(0.16)$ & 18.0424 & $(0.00)$ & 18.0424 & 19.7576 & $(0.25)$ & 19.7068 & $(-0.01)$ & 19.7083 \\
\hline SCSC & 32.3753 & $(0.13)$ & 32.3324 & $(0.00)$ & 32.3328 & 44.0880 & $(0.11)$ & 44.0351 & $(-0.01)$ & 44.0404 \\
\hline SCSF & 9.3224 & $(0.18)$ & 9.3057 & $(0.00)$ & 9.3058 & 6.6576 & $(0.21)$ & 6.6431 & $(0.00)$ & 6.6434 \\
\hline SCSS & 26.2909 & $(0.89)$ & 26.0755 & $(0.06)$ & 26.0598 & 36.2924 & $(0.25)$ & 36.2020 & $(0.00)$ & 36.2020 \\
\hline SFSF & 8.2593 & $(0.22)$ & 8.2410 & $(0.00)$ & 8.2412 & 6.0182 & $(0.16)$ & 6.0085 & $(0.00)$ & 6.0087 \\
\hline SSFF & 2.2628 & $(7.31)$ & 2.1195 & $(0.52)$ & 2.1086 & 1.9831 & (15.17) & 1.7399 & $(1.05)$ & 1.7219 \\
\hline SSSF & 9.0843 & $(0.52)$ & 9.0395 & $(0.03)$ & 9.0372 & 6.5108 & $(0.63)$ & 6.4721 & $(0.03)$ & 6.4702 \\
\hline SSSS & 22.4097 & $(1.88)$ & 22.0258 & $(0.14)$ & 21.9952 & 31.4656 & $(3.74)$ & 30.4147 & $(0.28)$ & 30.3310 \\
\hline SFFF & 3.5083 & $(0.03)$ & 3.5066 & $(-0.01)$ & 3.5071 & 1.4845 & $(-0.02)$ & 1.4845 & $(-0.02)$ & 1.4848 \\
\hline
\end{tabular}


Table 4

Effect of material orthotropy on first nondimensional frequency and compressive buckling force. Square plate with one ply at 45 . Results obtained using CLT and $50 \times 50$ functions.

\begin{tabular}{|c|c|c|c|c|c|c|c|c|c|c|}
\hline & \multirow[t]{2}{*}{$E_{1} / E_{2}$} & \multicolumn{3}{|c|}{ CCSS } & \multicolumn{3}{|c|}{ SSSS } & \multicolumn{3}{|c|}{ SSFF } \\
\hline & & Ritz & Abaqus S4R & $\%$ diff & Ritz & Abaqus S4R & $\%$ diff & Ritz & Abaqus S4R & $\%$ diff \\
\hline \multirow[t]{4}{*}{$\bar{\omega}_{1}$} & 73.36 & 30.6645 & 30.5823 & 0.27 & 22.1228 & 21.9952 & 0.58 & 2.1556 & 2.1086 & 2.23 \\
\hline & 40 & 24.3788 & 24.3496 & 0.12 & 17.7266 & 17.6809 & 0.26 & 1.9457 & 1.9302 & 0.80 \\
\hline & 20 & 19.0884 & 19.0818 & 0.03 & 13.9644 & 13.9543 & 0.07 & 1.7513 & 1.7486 & 0.15 \\
\hline & 10 & 15.2679 & 15.2672 & 0.00 & 11.1561 & 11.1547 & 0.01 & 1.5743 & 1.5736 & 0.04 \\
\hline \multirow[t]{4}{*}{$\bar{N}_{x}$} & 73.36 & 42.3478 & 42.1342 & 0.51 & 30.6813 & 30.3308 & 1.16 & 1.7999 & 1.7215 & 4.55 \\
\hline & 40 & 32.5627 & 32.4791 & 0.26 & 23.1637 & 23.0360 & 0.55 & 1.4696 & 1.4461 & 1.63 \\
\hline & 20 & 23.8467 & 23.8270 & 0.08 & 16.4563 & 16.4293 & 0.16 & 1.1914 & 1.1878 & 0.30 \\
\hline & 10 & 17.2488 & 17.2465 & 0.01 & 11.4683 & 11.4651 & 0.03 & 0.9597 & 0.9588 & 0.09 \\
\hline
\end{tabular}

Table 5

Benchmark solutions derived using $R \times R$ functions. Simply-supported plate with one ply oriented at $\theta$

\begin{tabular}{|c|c|c|c|c|c|c|}
\hline \multirow[b]{2}{*}{$\theta$} & \multicolumn{3}{|c|}{$\bar{\omega}_{1}$} & \multicolumn{3}{|c|}{$\bar{N}_{x}$} \\
\hline & 30 & 45 & 60 & 30 & 45 & 60 \\
\hline$E_{1} / E_{2}$ & & $R=100$ & & & $R=100$ & \\
\hline 73.36 & 22.7151 & 22.0258 & 22.7151 & 38.9076 & 30.4147 & 24.1977 \\
\hline 40 & 18.0025 & 17.6872 & 18.0025 & 26.8234 & 23.0524 & 19.3150 \\
\hline 20 & 14.0540 & 13.9539 & 14.0540 & 17.7112 & 16.4281 & 15.0587 \\
\hline 10 & 11.1989 & 11.1544 & 11.1989 & 11.8983 & 11.4641 & 11.7180 \\
\hline$E_{1} / E_{2}$ & & $R=150$ & & & $R=150$ & \\
\hline 73.36 & 22.7024 & 21.9934 & 22.7024 & 38.8649 & 30.3253 & 24.174 \\
\hline 40 & 17.9979 & 17.6753 & 17.9979 & 26.8080 & 23.0188 & 19.307 \\
\hline 20 & 14.0530 & 13.9513 & 14.0530 & 17.7082 & 16.4209 & 15.057 \\
\hline 10 & 11.1988 & 11.1540 & 11.1988 & 11.8980 & 11.4633 & 11.717 \\
\hline$E_{1} / E_{2}$ & & $R=200$ & & & $R=200$ & \\
\hline 73.36 & 22.6964 & 21.9772 & 22.6964 & 38.8446 & 30.2806 & 24.1635 \\
\hline 40 & 17.9959 & 17.6698 & 17.9959 & 26.8011 & 23.0031 & 19.3037 \\
\hline 20 & 14.0526 & 13.9502 & 14.0526 & 17.7070 & 16.4179 & 15.0566 \\
\hline 10 & 11.1987 & 11.1539 & 11.1987 & 11.8979 & 11.4630 & 11.7176 \\
\hline$E_{1} / E_{2}$ & & $R=250$ & & & $R=250$ & \\
\hline 73.36 & 22.6929 & 21.9674 & 22.6929 & 38.8328 & 30.2538 & 24.1571 \\
\hline 40 & 17.9948 & 17.6666 & 17.9948 & 26.7974 & 22.9941 & 19.3018 \\
\hline 20 & 14.0524 & 13.9496 & 14.0524 & 17.7064 & 16.4163 & 15.0563 \\
\hline 10 & 11.1987 & 11.1538 & 11.1987 & 11.8978 & 11.4628 & 11.7176 \\
\hline
\end{tabular}

where various kinematic theories are adopted. FSDT is applied by considering two different values of the shear factor. In the first case, the shear factor is taken unitary; in the second case, denoted with FSDT*, the shear factor is computed by adopting the technique implemented in the Abaqus S4-family elements [62]. For this specific case, the shear factor is equal to 0.7420 for the first layup, and 0.0677 for the second. In addition, the equivalent single layer theories $\mathrm{ED}_{332}$ and $\mathrm{ED}_{554}$ and the layerwise $\mathrm{LD}_{222}$ are employed. The comparison of the Ritz predictions using Legendre polynomials is presented against Abaqus 2D, using S4R elements, and 3D analyses. The latter are performed by making use of C3D8I elements, 8-node elements with incompatible modes. The mesh sizes, defined after a preliminary convergence study, are defined by considering:

- $100 \times 100$ elements along the planar directions, and 4 elements per ply for $a / h>10$

- $80 \times 80$ elements along the planar directions, and 8 elements per ply for $a / h \leqslant 10$

The choice is motivated by the prevalent role played by the transverse shear deformations as the ratio $a / h$ becomes smaller. It follows that thicker configurations, as it is the case for $a / h=5$ and 10 , need to be described with a higher through-the-thickness refinement. In this cases, the in-plane description is reduced to 80 elements to mitigate the computational burden.

As observed from Table 6, the plate response becomes increasingly dominated by the through-the-thickness behavior as the ratio $a / h$ is reduced. It is noted that the refinement along in-plane directions given by the increase of terms $R$ in the assumed Ritz series becomes less and less important, and what drives the accuracy of the solution is the enrichment of the assumed kinematic description rather than the adoption of more admissible functions. For example, for a thick plate with layup [45/-45/45] and $a / h=5$, the deviation of the Ritz prediction from the reference 3D analysis goes from approximately $10.7 \%$ to only $10.5 \%$ by changing $R$ from 10 to 60 when the plate is modeled according to FSDT. Instead, only 10 functions are needed to have an accuracy of approximately $1.6 \%$ when the theory is changed to $\mathrm{ED}_{554}$. Thus, in this case, the best tradeoff in terms of degrees of freedom is achieved by increasing the order of the theory while keeping relatively low the number of admissible functions. When moderately thick plates are analyzed, i.e. $a / h=25$, it is more difficult to find the optimal balance between number of trial functions and order of the theory. For this class of panels, the availability of a Ritz formulation capable of handling efficiently several degrees of freedom could be important to detect the model with the best accuracy-to-degrees-of-freedom ratio.

The results of Table 6 demonstrate also the advantages given by the use of FSDT with a shear factor computed referring to an advanced approach, as it is done in Abaqus. For instance, for the plates with layup [45/-45/45], the results obtained with FSDT* are very close to those available from high-order theories. However, the adoption of FSDT* can give very inaccurate predictions in the presence of elastic couplings associated with the plate non-symmetry, thus suggesting the adoption of higher-order theories not demanding for a shear factor evaluation. Indeed the approach adopted from Abaqus [62] to estimate the shear correction factor relies on the assumption that shell section directions are coincident with the principal bending directions. This is not the case for the considered anti-symmetric angle-ply layup, which is characterized by a membrane-twisting coupling. The shear factor obtained from the approach of Abaqus and employed in the FSDT* model is 0.0677 : this excessively low value underestimates the transverse shear stiffness and yields excessively low eigenfrequencies. Since high-order theories are capable of representing the through-thickness distribution of the transverse shear, they do not require shear correction factors and can provide more accurate results, as revealed by the percent differences with the 3D finite element solutions.

As done in the case of thin plates, a comparison is here presented for different choices of the admissible set when they are applied to the analysis of thick plate problems. The results are obtained with models based on $\mathrm{ED}_{332}$ theory and are plotted in Figs. 4 and 5 in terms of the first natural frequency and the compressive buckling load, respectively. In the first case, three lay-ups are considered, with plies at $[\theta],[ \pm \theta]$ and $[\theta /-\theta / \theta]$. The buckling response of the anti-symmetric layup $[\theta /-\theta]$ is non bifurcational and shall hence be omitted. The results are obtained by considering $R=S=30$ functions and are reported in terms of 
Table 6

First nondimensional frequencies $\bar{\omega}_{1}$ for SSSS square plates with different elastic couplings. (Note: ${ }^{\text {a }}$ used as reference for percent difference evaluation).

\begin{tabular}{|c|c|c|c|c|c|c|c|}
\hline Layup & $\mathrm{a} / \mathrm{h}$ & $\mathrm{R}$ & FSDT & FSDT* & $\mathrm{ED}_{332}$ & $\mathrm{ED}_{554}$ & $\mathrm{LD}_{222}$ \\
\hline \multirow[t]{12}{*}[45/-45/45]{} & 100 & 10 & $26.1231(1.73)$ & $26.0921(1.61)$ & $26.1046(1.66)$ & $26.0928(1.61)$ & $26.0899(1.60)$ \\
\hline & & 20 & $25.8567(0.69)$ & $25.8294(0.58)$ & $25.8290(0.58)$ & $25.7989(0.47)$ & $25.7871(0.42)$ \\
\hline & & 60 & $25.7573(0.30)$ & $25.7330(0.21)$ & $25.7174(0.15)$ & $25.6690(-0.04)$ & $25.6481(-0.12)$ \\
\hline & & & \multicolumn{5}{|c|}{ Abaqus C3D8I-S4R: $25.6794^{\mathrm{a}}-25.6980$} \\
\hline & 25 & 10 & $25.1194(3.15)$ & $24.8190(1.92)$ & $24.8958(2.24)$ & $24.7957(1.83)$ & $24.7655(1.70)$ \\
\hline & & 20 & $24.9276(2.37)$ & 24.6409 (1.19) & $24.6860(1.37)$ & $24.5533(0.83)$ & $24.5057(0.63)$ \\
\hline & & 60 & $24.8636(2.10)$ & $24.5823(0.95)$ & $24.6037(1.04)$ & $24.4498(0.40)$ & $24.3963(0.19)$ \\
\hline & & & \multicolumn{5}{|c|}{ Abaqus C3D8I-S4R: $24.3512^{\mathrm{a}}-24.5716$} \\
\hline & 5 & 10 & $15.9845(10.76)$ & $14.6610(1.59)$ & $14.9577(3.64)$ & $14.6661(1.62)$ & $14.6294(1.37)$ \\
\hline & & 20 & $15.9616(10.60)$ & $14.6454(1.48)$ & $14.9249(3.42)$ & $14.6282(1.36)$ & $14.5908(1.10)$ \\
\hline & & 60 & $15.9546(10.55)$ & $14.6407(1.45)$ & $14.9101(3.31)$ & $14.6112(1.24)$ & $14.5731(0.98)$ \\
\hline & & & \multicolumn{5}{|c|}{ Abaqus C3D8I-S4R: $14.4317^{\mathrm{a}}-14.6405$} \\
\hline \multirow[t]{12}{*}[\pm45]{} & 100 & 10 & $23.8062(0.92)$ & $23.3349(-1.07)$ & $23.7503(0.69)$ & $23.7072(0.50)$ & $23.7266(0.59)$ \\
\hline & & 20 & $23.8021(0.91)$ & $23.3296(-1.10)$ & $23.6882(0.42)$ & $23.6174(0.12)$ & $23.6443(0.24)$ \\
\hline & & 60 & $23.8012(0.90)$ & $23.3284(-1.10)$ & $23.6674(0.33)$ & $23.5932(0.02)$ & $23.6142(0.11)$ \\
\hline & & & \multicolumn{5}{|c|}{ Abaqus C3D8I-S4R: $23.5884^{\mathrm{a}}-23.3286$} \\
\hline & 25 & 10 & $23.2574(5.12)$ & $18.4353(-16.68)$ & $22.5929(2.11)$ & $22.2454(0.54)$ & $22.3838(1.17)$ \\
\hline & & 20 & $23.2521(5.09)$ & $18.4296(-16.70)$ & $22.5192(1.78)$ & $22.1676(0.19)$ & $22.2867(0.73)$ \\
\hline & & 60 & 23.2509 (5.09) & $18.4283(-16.71)$ & $22.5138(1.76)$ & $22.1610(0.16)$ & $22.2793(0.70)$ \\
\hline & & & \multicolumn{5}{|c|}{ Abaqus C3D8I-S4R: $22.1252^{\mathrm{a}}-18.4295$} \\
\hline & 5 & 10 & $16.1713(20.14)$ & $5.7214(-57.50)$ & $14.1054(4.79)$ & $13.5733(0.84)$ & $13.7696(2.30)$ \\
\hline & & 20 & $16.1670(20.11)$ & $5.7212(-57.50)$ & $14.0858(4.64)$ & $13.5536(0.69)$ & $13.7486(2.14)$ \\
\hline & & 60 & $16.1660(20.10)$ & $5.7211(-57.50)$ & $14.0768(4.58)$ & $13.5451(0.63)$ & $13.7390(2.07)$ \\
\hline & & & \multicolumn{5}{|c|}{ Abaqus C3D8I-S4R: $13.4606^{\mathrm{a}}-5.7220$} \\
\hline
\end{tabular}

percent differences between the values obtained by using the extended trigonometric shape functions and those computed by means of Legendre polynomials.

As observed from Fig. 4, the highest differences in the estimation of the fundamental frequency occur when $\theta=45$. For the symmetric layups, the errors due to the adoption of extended trigonometric functions tend to increase significantly for high values of $a / h$. This behavior is due to the poor performance of the trigonometric functions in describing the skewed pattern of the first mode shape associated with the plate flexural anisotropy. As the thickness increases, the dominant role played by the in-plane response is progressively replaced by the through-the-thickness behavior, which does not depend upon the admissible functions adopted. Despite the errors observed for thick plates are relatively smaller - thus the use of trigonometric function is not as detrimental as it is for thin plates -, it should be noticed that the errors can be as high as $2 \%$ when $a / h$ is equal to 10 . On the contrary, the antisymmetric configuration exhibits a response which is substantially independent of the plate thickness ratio. Indeed, the in-plane response does not present a complex skewed pattern, and is properly described even by trigonometric functions. It follows that no improvements are achieved when the through-the-thickness response becomes prevalent.

With regard to the buckling response, as reported in Fig. 5, the behaviour is similar to that observed for free vibrations. In this case, the magnitude of the errors is higher, and the relation between the two

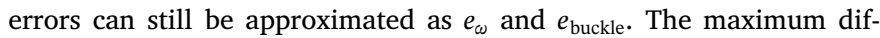
ference is observed for plates with $a / h=100$, and $\theta$ equal to 42 and 43 for the one- an three-ply configurations, respectively. The sharp drop of error observed for higher values of $\theta$ is due to a mode change from one to two halfwaves, which is predicted by the trigonometric functions but not by the Legendre ones. For clarity purposes, the reasons for these discrepancies are further investigated in Fig. 6, where the mode shapes are reported for a set of significant points associated with the one-ply configuration with $a / h=100$. Thus, apart from the effects associated with shifted buckling modes, the shape of the curve still resembles the one obtained for the free vibration problem.

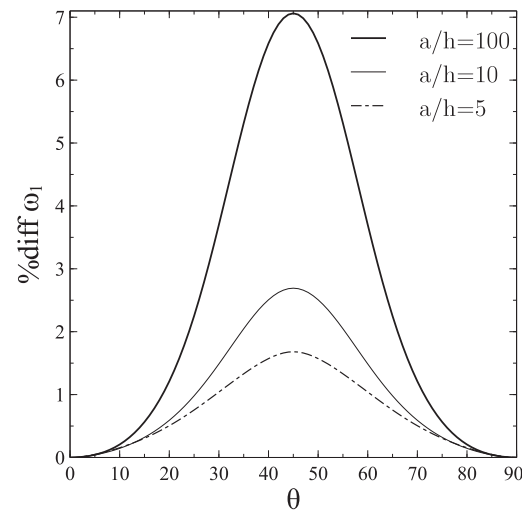

(a)

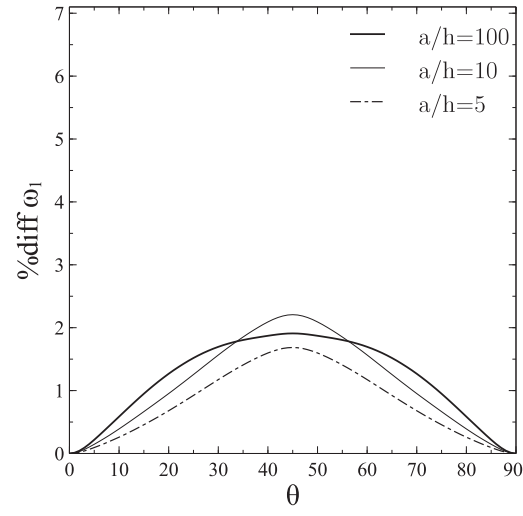

(b)

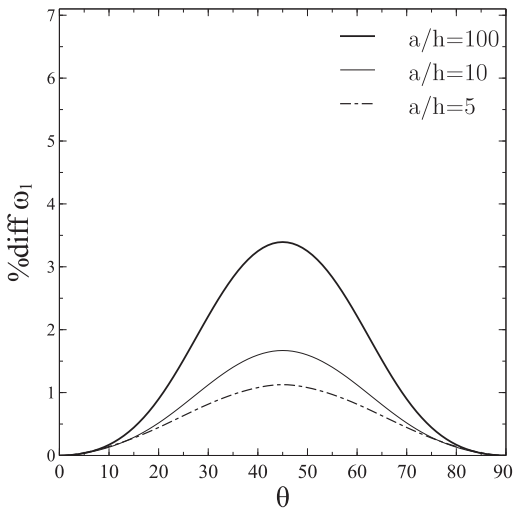

(c)

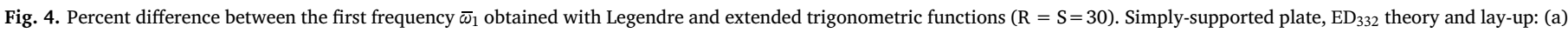
$[\theta]$, (b) $[ \pm \theta]$, (c) $[\theta /-\theta / \theta]$. 


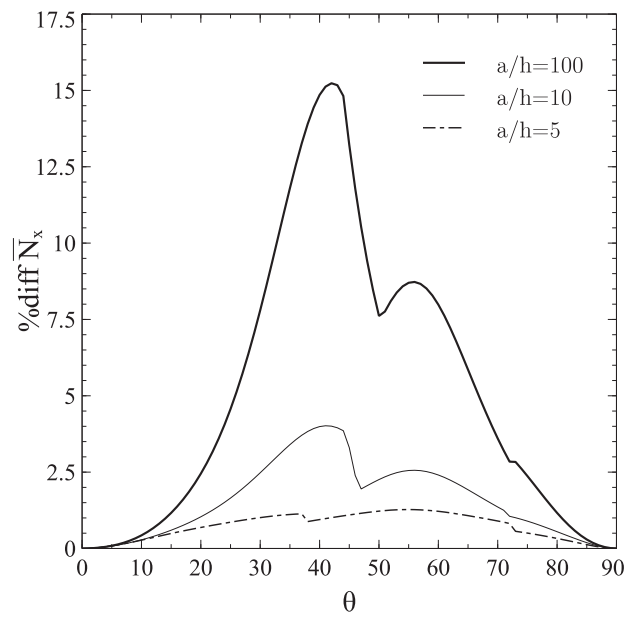

(a)

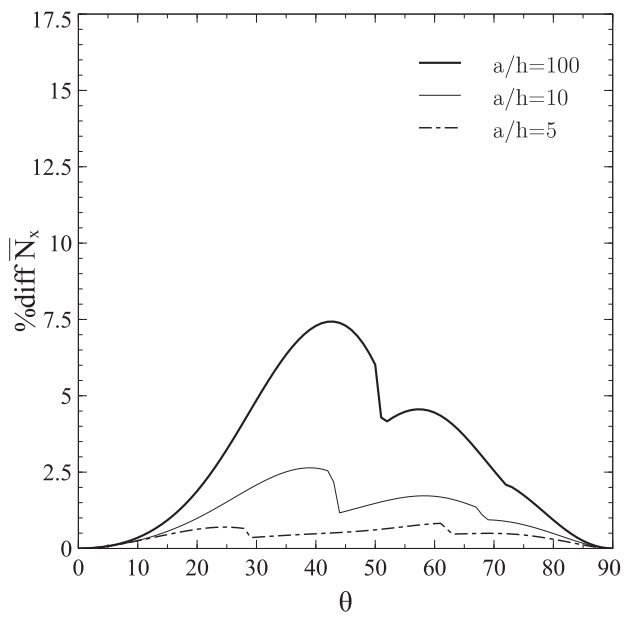

(b)

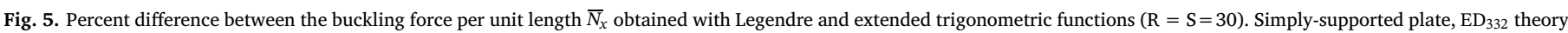
and lay-up: (a) $[\theta]$, (b) $[\theta /-\theta / \theta]$

\subsection{Remarks on the completeness of admissible functions}

An aspect that is sometimes overlooked when developing Ritz models is the completeness of the assumed basis. With this regard, it is useful to remind that completeness has to be defined with respect to the strain energy norm, which is, in general, different from the $L^{2}$ norm [48]. The energy norm involves the first derivatives - or, in the particular case of CLT, even the second ones - of the displacement components. The set of trial functions should then guarantee the ability of getting arbitrarily close to the true strain energy value.

A classical example where the completeness requirement is not fulfilled is encountered in the analysis of anisotropic simply-supported plates by means of the Navier-type expansion of Eq. (25). Whilst this set of functions satisfies the essential boundary conditions, the completeness is guaranteed in the $L^{2}$ norm, but not in the strain energy one. It follows that the displacement field cannot represent a constant deformation field and, unless special conditions are met, the solution cannot converge to the exact one. To clarify this aspect, an anti-symmetric plate with stacking $[ \pm 45]$ is considered. The plate is square and simply-supported at its four edges. The convergence of the first fundamental frequency as $R$ increases is reported in Fig. 7 for thin and thick plates, characterized by $a / h$ equal to 100 and 5 , respectively. The results are obtained by considering $\mathrm{ED}_{332}$ theory, and a comparison is provided for Ritz expansion based on Legendre polynomials and trigonometric functions in the form of Eqs. (25) and (26). It is observed that Legendre and extended trigonometric functions converge to the

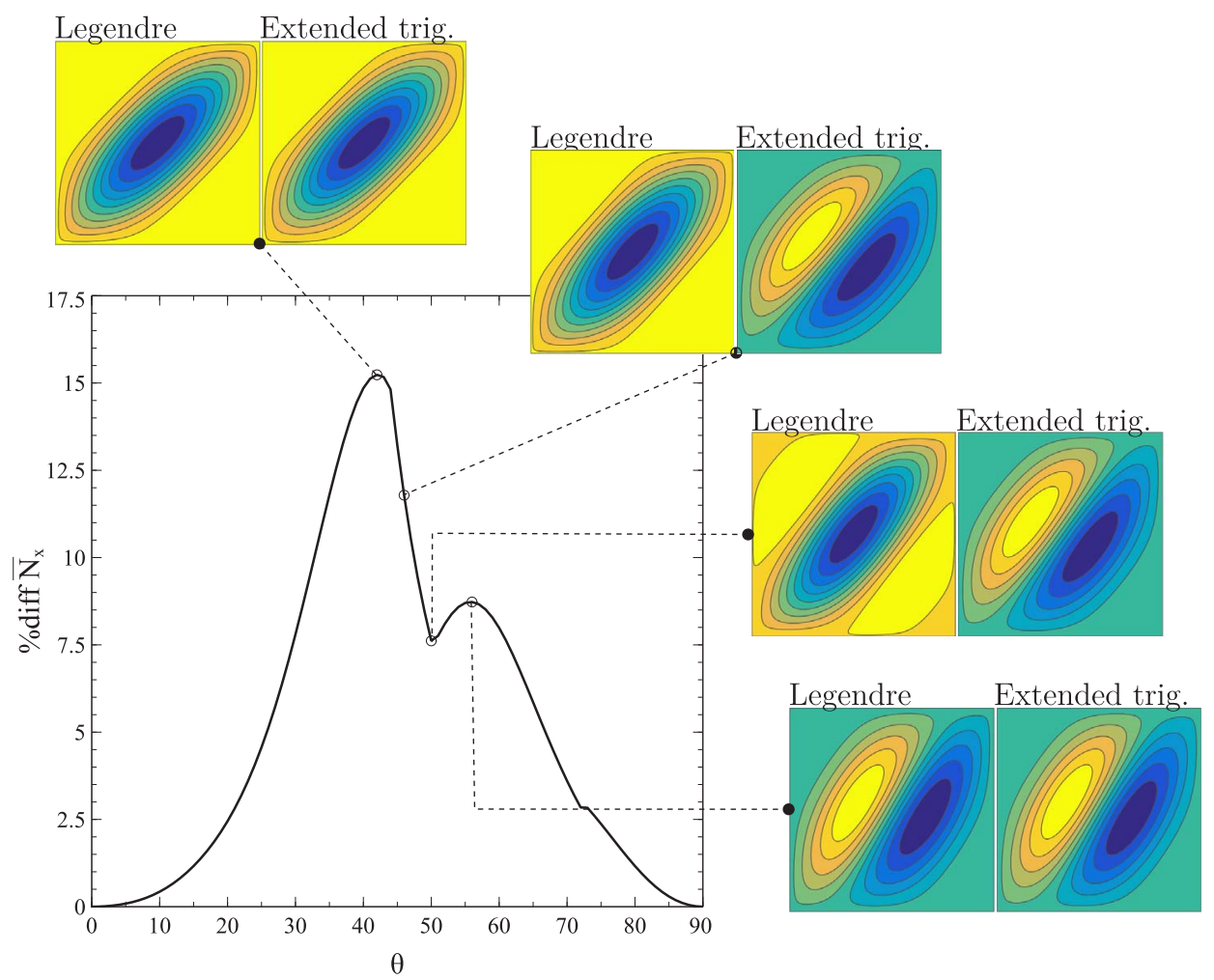

Fig. 6. Comparison of mode shapes predicted by Legendre and extended trigonometric functions for lay-up $[\theta]$ and $a / h=100$. 


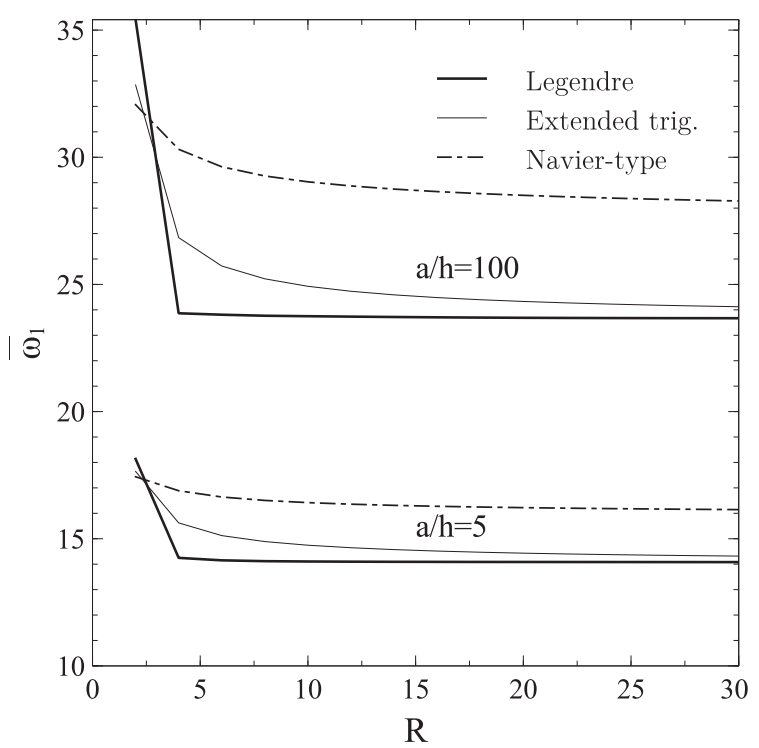

Fig. 7. Convergence of the solution for different sets of shape functions using $\mathrm{ED}_{332}$ theory. First fundamental frequency $\bar{\omega}_{1}$ of SSSS plate, with lay-up $[ \pm 45]$.

same solution, although with a different rate. On the contrary, the Navier-type solution tends asymptotically to an overstiff solution. This trend is more clear for thin plates, where the role played by in-plane behavior is predominant. In any case, the differences are not negligible even for the thick configuration. In this example, the errors are mainly related to the coupling between in-plane and out-of-plane response induced by the non-symmetry of the laminate. In fact, the bending deflections happen in conjunction with in-plane displacements, and the inability to describe a constant strain state has the effect of overconstraining the solution.

It is instructive to discuss a second example, which could appear as less intuitive, where the absence of polynomial contributions prevents convergence to correct results. For this purpose, a symmetric lay-up is considered, with one ply oriented at 45 . Two length-to-thickness ratios are analyzed in order to remark the role played by this parameter. The first nondimensional vibration frequency of the plate is plotted in Fig. 8, where the curves are relative to the solutions obtained using Legendre polynomials and trigonometric shape functions, with and without the linear polynomial terms (see Eqs. (25) and (26)). The results highlight a

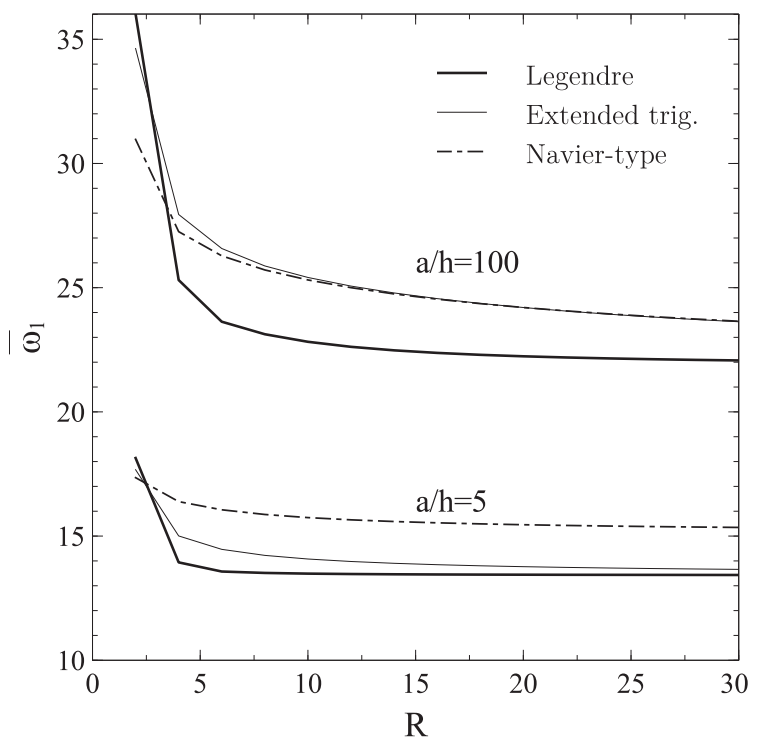

Fig. 8. Convergence of the solution for different sets of shape functions using $\mathrm{ED}_{332}$ theory. First fundamental frequency $\bar{\omega}_{1}$ of SSSS plate, with lay-up [45]. completely different mechanisms with respect to the previous example. In the case of thin plates, no distinction exists between the two trigonometric solutions. The convergence rate is very low due to the high amount of flexural anisotropy, but the frequency parameter converges to the correct solution, provided the number of functions is sufficiently high. In this case, the bending response is uncoupled from the membrane one, thus the inability to represent a constant strain has no strong impact on the results. On the contrary, the Navier-type functions lead to erroneous results when $a / h$ is reduced to 5 . Indeed, the behavior of thick plates is inherently three-dimensional, and the coupling between in-plane and out-of-plane deflections happens by means of the constitutive 3D law. It follows that the bending deflections promote the inplane displacements, whose description is lacking of the presence of a linear term. It is also observed that extended trigonometric functions converge much faster than in the case of thick plates. As pointed out previously, the issues related to the flexural anisotropy become less relevant for thick plates, as the through-the-thickness behavior gets more pronounced.

\subsection{Remarks on the numerical efficiency of different basis}

In previous examples, results associated with the generic wording orthogonal polynomials were computed by adopting Legendre polynomials as admissible functions. It was anticipated that identical values would have been obtained if Chebyshev polynomials were employed. An explicit comparison between the two polynomial sets is here provided for the four lay-ups L1 to L4 considered in Section 3.1.1.

Table 7 shows the first nondimensional frequency when the laminates have two sets of boundary conditions, fully simply-supported and fully clamped, and two different thickness ratios, $a / h=10$ and $a / h=100$. Thin plates are modeled according to FSDT, whereas the higher order theory $\mathrm{ED}_{332}$ is adopted to better represent the moderately thick configurations. A number of $30 \times 30$ polynomial functions is used in all the cases. As seen, all the values in Table 7 are identical. This outcome is in agreement with the findings of Ref. [14], where different sets of polynomial expressions were compared and demonstrated to lead to equal results, and it can be explained by recalling that the method of Ritz operates a projection of the exact solution onto the vector space spanned by the trial functions. In this case, the vector space spanned by Legendre and Chebyshev polynomials is the same, thus the solution is approximated with the same level of accuracy. Slight differences may occur if the in-plane integrals are computed numerically, as it is done in most of the implementations available in the literature. On the contrary, no differences can be appreciated whenever integration is performed exactly.

Despite the same degree of accuracy offered by the two set of functions, the computational efficiency is, in general, very different. In particular, noticeable differences regard the degree of sparsity of the resulting matrices, which, in turn, affects the time for computing the solution.

As an example, the percent number of nonzero terms in the stiffness

Table 7

First nondimensional frequencies $\bar{\omega}_{1}$ using different sets of orthogonal polynomials.

\begin{tabular}{lllllll}
\hline & & \multicolumn{2}{c}{$\mathrm{a} / \mathrm{h}=10, \mathrm{ED}_{332}$} & & \multicolumn{2}{c}{$\mathrm{a} / \mathrm{h}=100$, FSDT } \\
\cline { 3 - 4 } \cline { 6 - 7 } & & Legendre & Chebyshev & & Legendre & Chebyshev \\
\hline \multirow{2}{*}{ SSSS } & Layup L1 & 18.1320 & 18.1320 & & 22.0812 & 22.0812 \\
& Layup L2 & 13.4136 & 13.4136 & & 14.4961 & 14.4961 \\
& Layup L3 & 19.0494 & 19.0494 & & 23.8015 & 23.8015 \\
& Layup L4 & 15.1286 & 15.1286 & & 17.2967 & 17.2967 \\
\multirow{6}{*}{ CCCC } & & & & & \\
& Layup L1 & 24.2226 & 24.2226 & & 40.3987 & 40.3987 \\
& Layup L2 & 23.7414 & 23.7414 & & 30.8179 & 30.8179 \\
& Layup L3 & 22.8171 & 22.8171 & & 29.9020 & 29.9020 \\
& Layup L4 & 23.1717 & 23.1717 & & 31.3885 & 31.3885 \\
\hline
\end{tabular}


Table 8

Percent number of not-null terms in the stiffness matrix using analytical and numerical integration; $E D_{332}$ theory, $30 \times 30$ functions. ( ${ }^{\text {a }}$ using numerical integration).

\begin{tabular}{lllll}
\hline & & Legendre & Chebyshev & Extended trig. \\
\hline \multirow{2}{*}{ SSSS } & Layup L1 & $5.08\left(83.47^{\mathrm{a}}\right)$ & $39.22\left(83.47^{\mathrm{a}}\right)$ & 20.15 \\
& Layup L2 & $2.54\left(93.26^{\mathrm{a}}\right)$ & $23.41\left(96.13^{\mathrm{a}}\right)$ & 0.19 \\
& Layup L3 & $4.72\left(99.03^{\mathrm{a}}\right)$ & $37.57\left(99.99^{\mathrm{a}}\right)$ & 18.39 \\
& Layup L4 & $5.91\left(100.00^{\mathrm{a}}\right)$ & $47.35\left(100.00^{\mathrm{a}}\right)$ & 24.50 \\
& & & \\
CCCC & Layup L1 & $2.98\left(83.47^{\mathrm{a}}\right)$ & $39.88\left(83.47^{\mathrm{a}}\right)$ & $/$ \\
& Layup L2 & $1.83\left(96.64^{\mathrm{a}}\right)$ & $24.17\left(96.69^{\mathrm{a}}\right)$ & $/$ \\
& Layup L3 & $2.86\left(99.99^{\mathrm{a}}\right)$ & $38.22\left(100.00^{\mathrm{a}}\right)$ & $/$ \\
& Layup L4 & $3.60\left(100.00^{\mathrm{a}}\right)$ & $48.14\left(100.00^{\mathrm{a}}\right)$ & $/$ \\
\hline
\end{tabular}

matrix of the models based on $\mathrm{ED}_{332}$ is shown in Table 8. For the sake of completeness, in addition to Chebyshev and Legendre polynomials, the analysis now includes the models obtained using extended trigonometric functions. For the first two sets of functions, the results in the brackets illustrate the case where the integration is carried out numerically by employing Gauss quadrature with $R$ points along both directions. Instead, trigonometric functions are always integrated analytically due to the simplicity of the related expressions. As seen from Table 8, whenever integration is carried out analytically, Legendre polynomials are always associated with a higher degree of sparsity than Chebyshev ones. The difference is remarkable and so the impact on the computational cost. At the same time, the advantages of performing analytical integration of the in-plane integrals can be appreciated. Numerical integration determines the loss of any sort of sparsity - in some cases the stiffness matrix is completely full -, and no significant differences can be observed between different sets of orthogonal polynomials. Trigonometric functions, whose adoption is restricted to the simply-supported case, are generally less advantageous than Legendre polynomials. At the same time, the degree of sparsity is higher than for Chebyshev polynomials, although convergence is generally slower. In the case of layup L2, which is a cross-ply configuration, the orthogonality of sines and cosines is fully exploited, and the stiffness matrix is almost diagonal. This is a special case and, in the presence of any additional elastic coupling, the degree of sparsity offered by trigonometric functions is lost. This situation is further illustrated in Fig. 9, where the sparsity pattern of the stiffness matrix is reported for different functions and lay-ups, after performing integration in closed-form manner. As seen, the number of nonnull contributions is higher when the principal axis are aligned with off-axis directions, as it happens for the lay-up [45]. This observation holds independently of the kind of functions adopted. At the same time, the highest degree of sparsity offered by Legendre polynomials can be clearly appreciated.

Given the efficiency of the proposed implementation, it is interesting to provide an insight into the computational time required by typical calculations. A comparison is presented against the results of Moreno-Garcia et al. [23], where computational times are reported for different sets of shape functions. A fully clamped isotropic plate is taken as a benchmark; dimensions and material properties are those of Ref. [23]. The time needed to extract the first ten frequencies is reported in Fig. 10(a) using a CPU with $32 \mathrm{~GB}$ of RAM, and 4 Intel core i7 and at $4 \mathrm{GHz}$. The efficiency of the present Ritz approach can be observed by the maximum time taken for an analysis using 100 shape functions along both the directions, approximately equal to $0.75 \mathrm{~s}$. Conversely, the results reported in Ref. [23] using modified characteristic functions (MCF) and orthogonal polynomials (OP) require computational times which are several order of magnitudes higher. This discrepancy, partly due to the smaller amount of RAM memory used in the computations of Ref. [23], is believed to be mainly motivated by the analytical

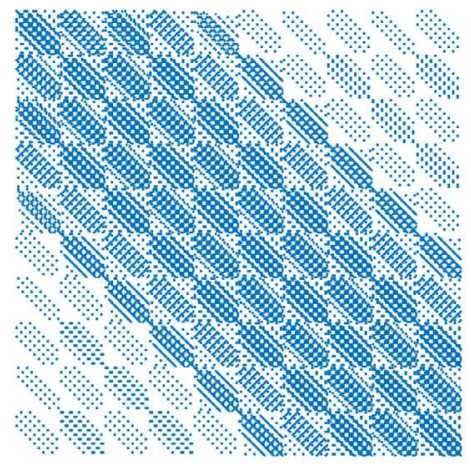

(a)

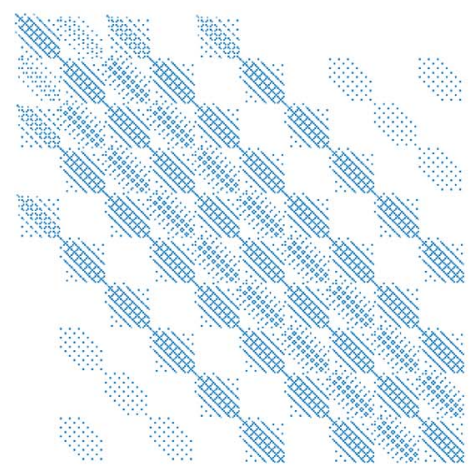

(d)

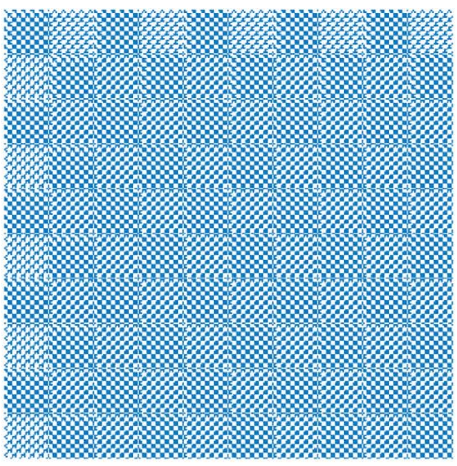

(b)

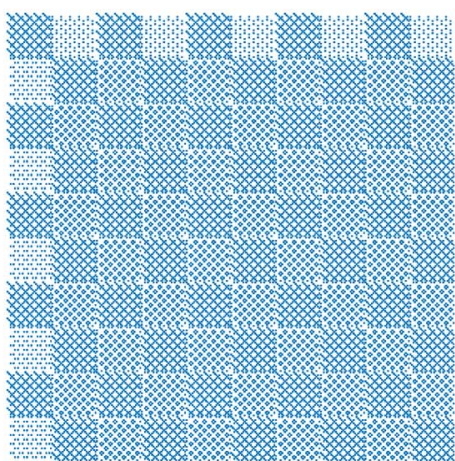

(e)

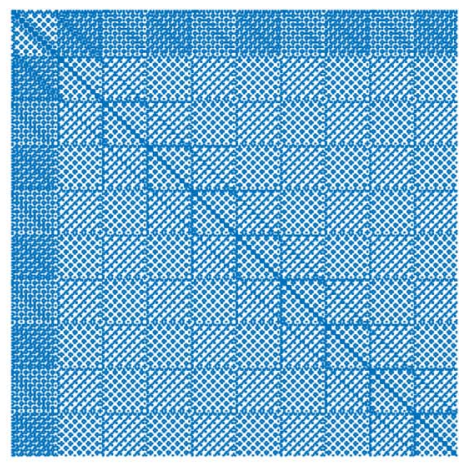

(c)

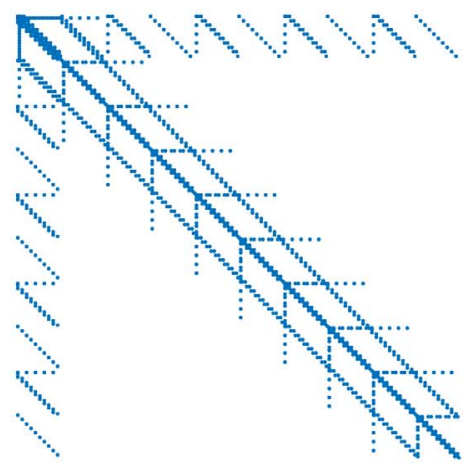

(f)

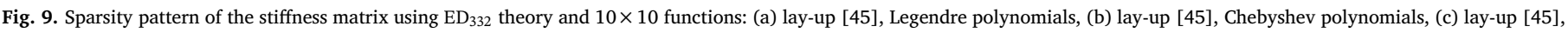
extended trigonometric functions, (d) lay-up [0/90], Legendre polynomials, (e) lay-up [0/90], Chebyshev polynomials, (f) lay-up [0/90], extended trigonometric functions. 


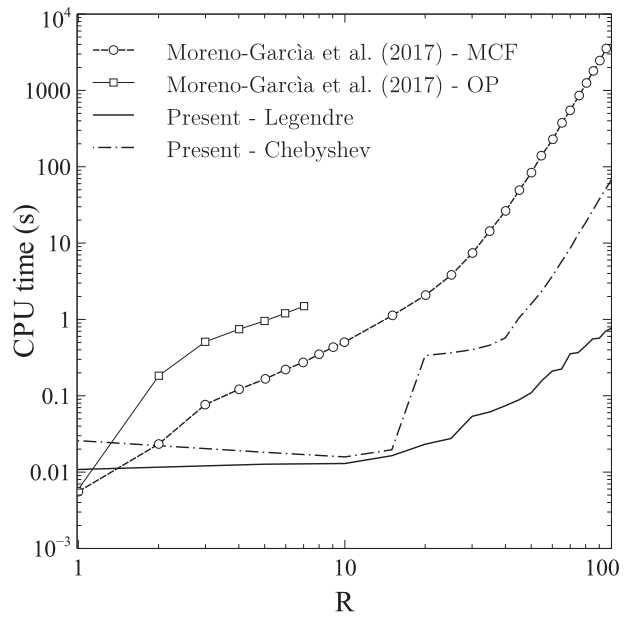

(a)

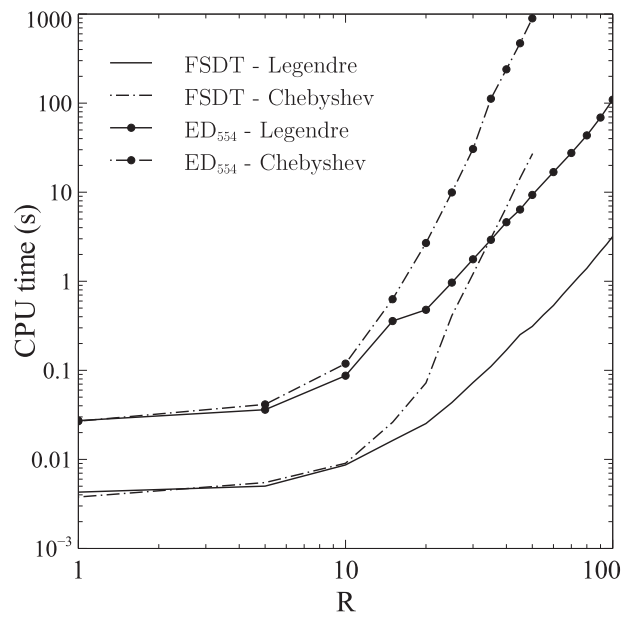

(b)

Fig. 10. Computational time against number of shape functions $R \times R$ : (a) CLT and comparison with Ref. [23], (b) FSDT and ED 554 .

integration here performed, and the subsequent advantages in terms of sparsity. At the same time, the results demonstrate the advantages of Legendre polynomials against Chebyshev ones. The same consideration holds in the case of higher-order theories, as reported in Fig. 10(b), where FSDT and $\mathrm{ED}_{554}$ theory are applied to the same test case. It can be noted that, using 100 Legendre functions along both directions, FSDT results are available in $3 \mathrm{~s}$, while $\mathrm{ED}_{554}$ in $109 \mathrm{~s}$. On the contrary, the time needed by Chebyshev polynomials is several orders of magnitudes higher.

Worth of mention is the fact that no numerical instabilities are encountered as the number of functions is increased. This was verified up to 100 functions in Fig. 10, and even to 250 functions in Table 5. This is in contrast with some results in the literature where the onset of complex eigenvalues is claimed, see for instance Ref. [23], or ill-conditioning restrictions are detected [29].

\section{Conclusions}

This work has discussed aspects related to the application of the Ritz method to buckling and free vibration analysis of highly anisotropic thin and thick plates. An implementation of the method is specifically developed with the aim of obtaining high computational efficiency. In so doing, problems with high degree of anisotropy, for which the convergence of the solution is particularly hard to achieve, can be analyzed using a large number of trial functions, leading to an unprecedented level of accuracy.

A set of exemplary results has been first presented for thin plates, showing that the most detrimental effects on the convergence of the solution are due to flexural anisotropy. This is particularly true in the presence of natural boundary conditions, such as simply-supported and free edges. Novel refined upper-bound solutions are provided for the most challenging cases, using up to 250 admissible functions along each in-plane direction. It is worth noting that no ill-conditioning issues nor numerical instabilities have been detected.

In the case of thick plates, the effects of flexural anisotropy are mitigated by the increasing role played by the through-the-thickness response. As the width-to-thickness ratio decreases, it has been shown that the accuracy of the solution is driven by the enrichment of the kinematic description more than the increase in the number of trial functions. Therefore, a variable-kinematic formulation capable of representing plate theories of increasing refinement within an unified modeling framework can be particularly convenient to preliminarily perform a tuning of the model to be used in the analysis.

An extensive comparison among different sets of admissible functions has been also presented. Overall, the results indicate the superiority of orthogonal polynomials with respect to trigonometric functions. This is true for all the cases, apart from those for which an exact Navier-type solution is available.

The advantages of adopting orthogonal polynomials are more relevant for thin plates, especially in the presence of strong flexural anisotropy. However, their use is suggested also for thick plates if accurate solutions are sought. Among the class of orthogonal polynomials, it was shown that identical results are obtained if Legendre and Chebyshev polynomials are adopted. In spite of that, a drastic difference exists in terms of computational efficiency as far as Legendre polynomials are responsible for a higher degree of sparsity. This feature is properly exploited only if in-plane integration is carried out analytically.

\section{References}

[1] Ritz W. Theorie der transversalschwingungen einer quadratische platte mit freien randern. Ann Phys-Leipzig 1909;333(4):737-86.

[2] Simons DA, Leissa AW. Vibrations of rectangular cantilever plates subjected to inplane acceleration loads. J Sound Vib 1971;17(3):407-22.

[3] Bassily SF, Dickinson SM. Buckling and lateral vibration of rectangular plates subject to inplane loads-a Ritz approach. J Sound Vib 1972;24(2):219-39.

[4] Leissa AW. The free vibration of rectangular plate. J Sound Vib 1973;31(3):257-93.

[5] Bassily SF, Dickinson SM. On the use of beam functions for problems of plates involving free edges. J Appl Mech 1975;42(4):858-64.

[6] Bhat RB. Natural frequencies of rectangular plates using characteristic orthogonal polynomials in Rayleigh-Ritz method. J Sound Vib 1985;102(4):493-9.

[7] Dickinson SM, Li EKH. On the use of simply supported plate functions in the Rayleigh-Ritz method applied to the flexural vibration of rectangular plates. J Sound Vib 1982;80(2):292-7.

[8] Cupial P. Calculation of the natural frequencies of composite plates by the RayleighRitz method with orthogonal polynomials. J Sound Vib 1997;201(3):385-7.

[9] Smith S, Bradford MA, Oehlers DJ. Numerical convergence of simple and orthogonal polynomials for the unilateral plate buckling problem using the Rayleigh-Ritz method. Int J Numer Meth Eng 1999;44(11):1685-707.

[10] Liew KM, Lam KY, Chow ST. Free vibration analysis of rectangular plates using orthogonal plate function. Comput Struct 1990;34(1):79-85.

[11] Baharlou B, Leissa AW. Vibration and buckling of generally laminated composite plates with arbitrary edge conditions. Int J Mech Sci 1987;29(8):545-55.

[12] Liew KM, Wang CM. pb-2 Rayleigh-Ritz method for general plate analysis. Eng Struct 1993;15(1):55-60.

[13] Saadatpour MM, Azhari M, Bradford MA. Analysis of general quadrilateral orthotropic thick plates with arbitrary boundary conditions by the Rayleigh-Ritz method. Int J Numer Meth Eng 2002;54(7):1087-102.

[14] Brown RE, Stone MA. On the use of polynomial series with the Rayleigh-Ritz method. Compos Struct 1997:39(3-4):191-6.

[15] Oosterhout GM, Hoogt PJM, Spiering RMEJ. Accurate calculation methods for natural frequencies of plates with special attention to the higher modes. J Sound Vib 1995;183(1):33-47.

[16] Mikhlin SG. The Numerical Performance of Variational Methods. The Netherlands: Wolters-Noordhoff Publishing Groningen; 1971.

[17] Budiansky B, Hu PC. The Lagrangian multiplier method of finding upper and lower limits to critical stresses of clamped plates. Report 848, NACA; 1946. 
[18] Narita Y, Leissa AW. Buckling studies for simply supported symmetrically laminated rectangular plates. Int J Mech Sci 1990;32(11):909-24.

[19] Vescovini R, Bisagni C. Single-mode solution for post-buckling analysis of composite panels with elastic restraints loaded in compression. Compos Part B: Eng 2012;43(3):1258-74.

[20] Vescovini R, Bisagni C. Two-step procedure for fast post-buckling analysis of composite stiffened panels. Comput Struct 2013;128:38-47.

[21] Beslin O, Nicolas J. A hierarchical functions set for predicting very high order plate bending modes with any boundary conditions. J Sound Vib 1997;202:633-55.

[22] Dozio L. On the use of the trigonometric Ritz method for general vibration analysis of rectangular Kirchhoff plates. Thin-Walled Struct 2011;49:129-44.

[23] Moreno-Garća P, Araújo dos Santos JV, Lopes H. A review and study on Ritz method admissible functions with emphasis on buckling and free vibration of isotropic and anisotropic beams and plates. Arch Computat Methods Eng 2017:1-31.

[24] Stone MA, Chandler HD. Errors in double sine series solutions for simply supported symmetrically laminated plates. Int J Mech Sci 1996;38(5):517-26.

[25] Yuan WX, Dawe DJ. Overall and local buckling of sandwich plates with laminated faceplates, Part II: Applications. Comput Methods Appl Mech Eng 2001;190(40):5215-31.

[26] Rao KM. Buckling analysis of anisotropic sandwich plates faced with fiber-reinforced plastics. AIAA J 1985;23(8):1247-53.

[27] Kim CG, Hong CS. Buckling of unbalanced anisotropic sandwich plates with finite bonding stiffness. AIAA J 1988;26(8):982-8.

[28] Gawandi A, Whitney JM, Brockman RA. Natural boundary conditions in the bending of anisotropic laminated plates. Compos Struct 2008;82(2):201-8.

[29] Wu Z, Raju G, Weaver PM. Comparison of variational, differential quadrature, and approximate closed-form solution methods for buckling of highly flexurally anisotropic laminates. J Eng Mech 2012;139(8):1073-83.

[30] Carrera E, Fazzolari FA, Demasi L. Vibration analysis of anisotropic simply supported plates by using variable kinematic and Rayleigh-Ritz method. J Vib Acoust 2011;133(6). 061017-1-061017-16.

[31] Fazzolari FA, Carrera E. Advanced variable kinematics Ritz and Galerkin formulations for accurate buckling and vibration analysis of anisotropic laminated composite plates. Compos Struct 2011;94(1):50-67.

[32] Fazzolari FA, Carrera E. Thermo-mechanical buckling analysis of anisotropic multilayered composite and sandwich plates by using refined variable-kinematics theories. J Therm Stresses 2013;36(4):321-50.

[33] Fazzolari F, Carrera E. Accurate free vibration analysis of thermo-mechanically pre/ post-buckled anisotropic multilayered plates based on a refined hierarchical trigonometric Ritz formulation. Compos Struct 2013;95:381-402.

[34] Fiedler L, Lacarbonara W, Vestroni F. A generalized higher-order theory for multilayered, shear-deformable composite plates. Acta Mech 2010;209:85-98.

[35] Dozio L, Carrera E. A variable kinematic Ritz formulation for vibration study of quadrilateral plates with arbitrary thickness. J Sound Vib 2011;330(18):4611-32.

[36] Dozio L, Carrera E. Ritz analysis of vibrating rectangular and skew multilayered plates based on advanced variable-kinematic models. Compos Struct 2012;94(6):2118-28.

[37] Vescovini R, Dozio L. A variable-kinematic model for variable stiffness plates: vibration and buckling analysis. Compos Struct 2016;142:15-26.

[38] D'Ottavio M, Dozio L, Vescovini R, Polit O. Bending analysis of composite laminated and sandwich structures using sublaminate variable-kinematic Ritz models. Compos Struct 2016;155:45-62.

[39] Vescovini R, D'Ottavio M, Dozio L, Polit O. Thermal buckling response of laminated and sandwich plates using refined 2-d models. Compos Struct 2017;176:313-28.
[40] Woodcock RL. Free vibration of advanced anisotropic multilayered composites with arbitrary boundary conditions. J Sound Vib 2008:312(4):769-88.

[41] Dozio L. Refined 2-D theories for free vibration analysis of annular plates: unified Ritz formulation and numerical assessment. Comput Struct 2015;147:250-8.

[42] Demasi L. $\infty^{3}$ hierarchy plate theories for thick and thin composite plates: the Generalized Unified Formulation. Compos Struct 2008;84(3):256-70.

[43] Demasi L. $\infty^{6}$ mixed plate theories based on the Generalized Unified Formulation. Part I: governing equations. Compos Struct 2009;87(1). 1-1.

[44] Carrera E. A class of two-dimensional theories for anisotropic multilayered plates analysis. Atti Accademia delle Scienze di Torino. Memorie Scienze Fisiche 1995;19:1-39.

[45] Carrera E. Theories and finite elements for multilayered, anisotropic, composite plates and shells. Arch Comput Methods Eng 2002;9(2):87-140.

[46] Carrera E, Ciuffreda A. A unified formulation to assess theories of multilayered plates for various bending problems. Compos Struct 2005;69(3):271-93.

[47] Monterrubio LE, Ilanko S. Proof of convergence for a set of admissible functions for the Rayleigh-Ritz analysis of beams and plates and shells of rectangular planform. Comput Struct 2015;147:236-43.

[48] Storch J, Strang G. Paradox lost: natural boundary conditions in the Ritz-Galerkin method. Int J Numer Methods Eng 1988;26(10):2255-66.

[49] Green AE. Double Fourier series and boundary value problems. Math Proc Cambridge Philos Soc 1944;40(3):222-8.

[50] Whitney JM, Leissa AW. Analysis of a simply supported laminated anisotropic rectangular plate. AIAA J 1970;8:28-33.

[51] Whitney JM. On the analysis of anisotropic rectangular plates. Technical Report AD-776 017. Air Force Materials Laboratory Wright-Patterson Air Force Base; 1972

[52] Malekzadeh K, Khalili MR, Jafari A, Mittal RK. Dynamic response of in-plane prestressed sandwich panels with a viscoelastic flexible core and different boundary conditions. J Compos Mater 2006;40(16):1449-69.

[53] Fantuzzi N, Tornabene F, Bacciocchi M, Neves A, Ferreira AJM. Stability and ac curacy of three Fourier expansion-based strong form finite elements for the free vibration analysis of laminated composite plates. Int J Numer Meth Eng 2017;111:354-82.

[54] Weaver PM. Approximate analysis for buckling of compression loaded long rectangular plates with flexural/twist anisotropy. Proc R Soc London A: Math Phys Eng Sci 2006;462(2065):59-73.

[55] Lanhe W, Hua L, Daobin W. Vibration analysis of generally laminated composite plates by the moving least squares differential quadrature method. Compos Struct 2005;68(3):319-30.

[56] Zhu Q, Wang X. Free vibration analysis of thin isotropic and anisotropic rectangular plates by the discrete singular convolution algorithm. Int J Numer Meth Eng 2011;86(6):782-800.

[57] Wang X, Wang Y, Xu S. DSC analysis of a simply supported anisotropic rectangular plate. Compos Struct 2012;94(8):2576-84.

[58] Hyer MW. Stress analysis of fiber-reinforced composite materials. New York: McGraw-Hill; 1998.

[59] Jones RM. Mechanics of composite materials. CRC Press; 1998.

[60] Reddy JN. Mechanics of laminated composite plates and shells: theory and analysis Boca Raton: CRC Press; 2004.

[61] Wang JTS. On the solution of plates of composite materials. J Compos Mater 1969;3(3):590-2.

[62] Abaqus, version 6.16. User's manual. SIMULIA World Headquarters, Providence, RI, USA; 2016. 